Int. J. Speleol. 10 (1978), pp. 113 - 155

\title{
Contribution à l'étude des cavités karstiques du Djurdjura (Algérie). Description morpho- hydrogéologique et cadre évolutif
}

par

YVES QUINIF*

Contribution to the study of Karstik caves of Djurdjura (Algeria). Morpho-hydrogeological description and evolutive synthesis.

SUMMARY

In North-Africa, the karst of Djurdjura Mountains is important because it shows high-alpine characters. In others papers, we have studied shallow morphology and speleological explorations. Here, we describe the caves: morphology, qualitative hydrology and fillings. These cavities are replaced in their morpho-structural context. We make distinctions between kinds of cavities. Gulfs and resurgences characterize high-alpine karst which is actual.Other caves which have their opening at the middle of slopes are dry, disconnected of actual morphological context. They belong to past karstification phases. From the synthesis of those elements, we show that it is possible to use karstic data in the reconstruction of morpho-structural evolution of a country.

\section{INTRODUCTION.}

La chaîne du Haut-Djurdjura occupe par ses caractères morpho-structuraux et climatiques une position bien particulière en Algérie et, d'une façon générale, en Afrique du Nord. Le Djurdjura, le Haut-Atlas, le Rif marocain et quelques massifs constantinois (Quinif, 1976a; Coiffait, Quinif , Vila, 1975) constituent les karsts de type haut-alpin les plus importants en Afrique.

Compte tenu d'une extension spatiale peu importante, le karst du Djurdjura est riche et diversifié. C'est le caractère haut-alpin qui le marque le plus, à la fois à l'air libre et dans les cavités. La morphologie de surface est typique et même exceptionnelle (Quinif, 1976a; Quinif, 1977): champs de lapiés, dolines, poljés possedant des caractères propres. Les cavités, les circulations souterraines, ainsi que les phénomènes connexes achèvent de prouver, si besoin était, l'intérêt karstologique du Djurdjura. L'aspect purement spéléologique a été

* Faculté Polytechnique de Mons, département de minéralogie-pétrographie. Adresse personnelle: rue de la place, 30, B 7401 - Naast, Belgique 
abordé dans de nombreux articles (Belin, 1941; Belin, 1948; Birebent, 1948; Birebent, 1953; Quinif, 1973; Quinif, 1975; Quinif, 1976b).

Vu que les principaux traits de la morphologie karstique du Djurdjura ont été exposés ailleurs, nous nous intéresserons surtout ici aux cavités et à leur contexte. Nous verrons d'abord le cadre géographique, géologique et climatique. Suivra un rapide rappel des formes karstiques aériennes. Ensuite, nous passerons en revue les cavités sous l'angle morpho-hydrogéologique en relation avec les formes extérieures. Une première classification des cavités apparaîtra. Il sera nécessaire de les replacer dans le contexte général du réseau. La dernière partie sera consacrée à quelques points concernant l'evolution générale du massif particulièrement éclairés par les phénomènes karstiques.

Toutes les observations utilisées proviennent évidemment des cavités explorées et visitées. Nous n'avons pas le sentiment d'avoir épuisé le potentiel spéléologique du massif!, même dans la conception d'une première approche.

Ceci m'amène à remercier tous ceux qui ont permis la réalisation de cette étude. Je songe en particulier à mon épouse Annie, à Martine et Michel Boucq, Claude Vandecatsye et Jean-Louis Daussogne de l'Equipe Spéléo du Centre, Daniel Hissung du spéléo-club de la M.J.C. de la Valette, à notre ami à tous François Delhez, trop tôt enlevé à notre amitié. Que les autres compagnons m'excusent de ne pas les citer tous ici: la liste serait trop longue; qu'ils soient sûrs que je ne les oublie pas. J'aurai une pensée particulière pour tous mes amis algériens: les spéléos de Béjaia, Hanafi et Mohammed, ainsi que les co-

Fig.1. Localisation du Djurdjura et aspect général de la chaîne.

A: Djebel Kouriet; il est essentiellemęnt formé de calcaires nummulitiques.

B: Djebel Tiassassine; avec son point culminant à $2102 \mathrm{~m}$.

C: Djebel Tarzou bou Arous.

D: Djebel Haïer.

E: Djebel Timédouïne.

F: Azérou ou Gougane.

G: Azérou Thaltat.

$\mathrm{H}$ : Djebel Tigounatine.

I: Terga Iroumienne (notée parfois Terga m'ta Roumi).

$\mathrm{J}$ : Adrar Timésouïne.

K: Azérou Tidjer.

L: Tamgout de Lala Khedidja.

M: Massif d'Aït Ouabane.

N: Massif de Tirourda.

O: Région de Tizi Boussouill; aggrandi à la figure 3 .

1. Agounis de la Dent du Lion avec le Tesserft de la Bérézina.

2. Tessereft Hamid.

3. Ifri Delhez.

4. Takouaz Guérissène.

5. Anou Thinichiguine (nommé aussi Anou Tenchiji).

6. Ifri Entmgharthe.

7. Aïn Tinzert.

8. Sources de Merkalla; ces deux émergences sont les deux sorties d'eau principales de l'Haïzer.

9. Ansort Arbaïloune.

10. Aïn-el-Hammam; résurgence des eaux de l'Anou Boussouiïl.

11. Ansor Aberkane et Ansor Lekhlat. 


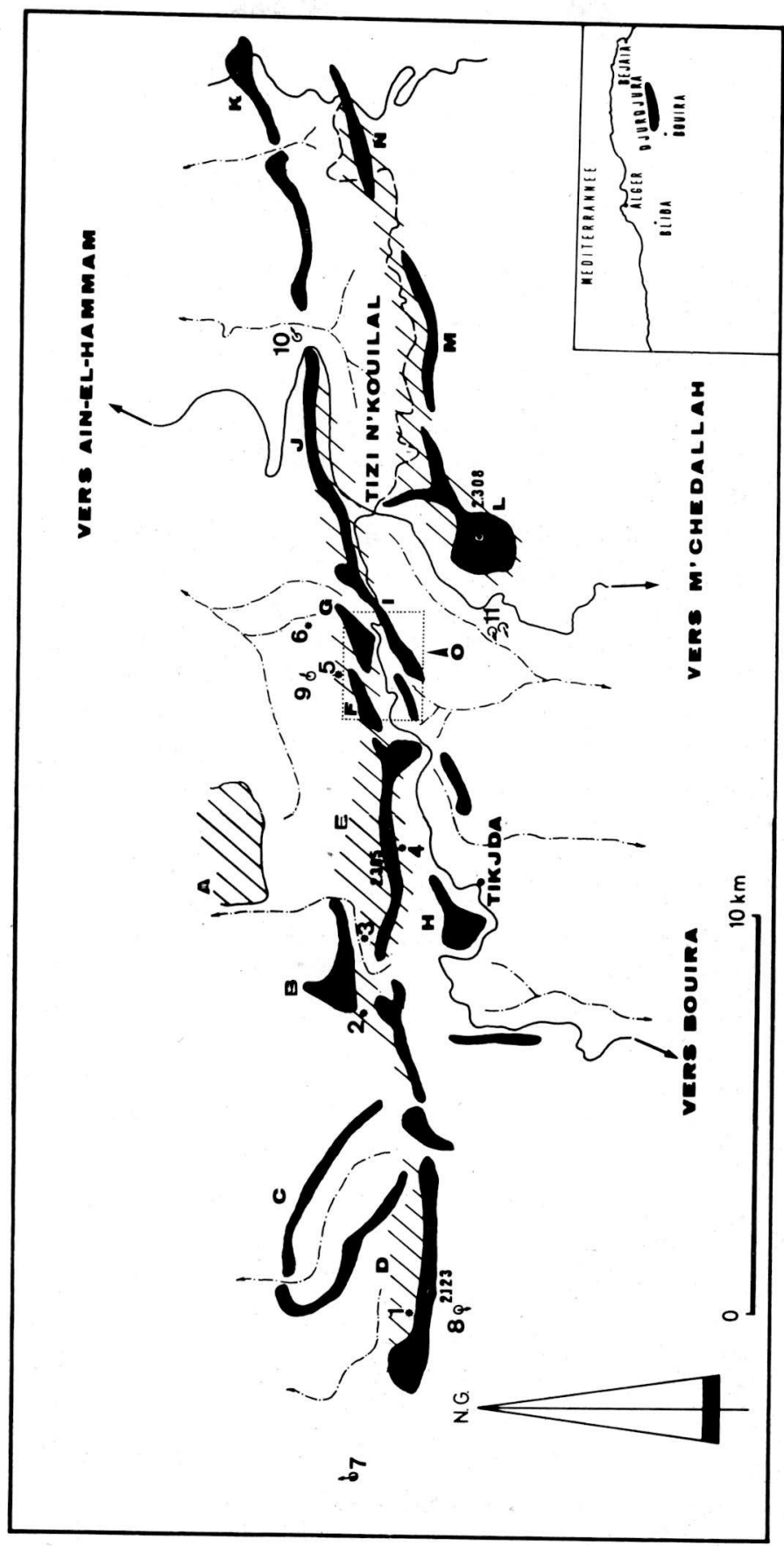


pains de Timghras: Messaoud et Boussad. Merci à tous et qu'ils trouvent ici l'expression de nos communes activités en ces 5 années.

\section{LE MILIEU GÉOGRAPHIQUE ET GÉOLOGIQUE.}

\section{A. Aspects géographiques.}

Le Djurdjura est une chaîne montagneuse située à $150 \mathrm{~km}$ au S-E d'Alger (fig. 1). Longue de $40 \mathrm{~km}$, large au maximum d'une dizaine de $\mathrm{km}$, elle culmine au Lala Khedidja $(2308 \mathrm{~m})$. La chaîne est formée de hautes crêtes parallèles entr'elles s'allongeant d'ouest. Correspondant aux grandes masses calcaires, elles dominent de leurs $2000 \mathrm{~m}$ un ensemble de massifs aux formes rondes et d'atitude inférieure à $1000 \mathrm{~m}$ : roches sédimentaires détritiques et métamorphiques tendres de la Grande Kabylie.

Classiquement, on divise le Djurdjura en 4 zones (Flandrin, 1952): les chaînons septentrionaux articulés sur le Pic du Pressoir (Tizi Guessig, $2102 \mathrm{~m}$ ), les grosses masses karstifiées: Haïzer, Ras Timédouïne, Azérou ou Gougane, Azérou Thaltat, de longues crêtes étroites, également karstifiées: Ras Tigounatine, Terga Iroumienne, Adrar Timésouïne, Azérou Tidjer et, enfin, occupant une position méridionale, l'imposante pyramide de Lala Khedidja, prolongée à l'est par l'Azérou Madène et le massif de Tirourda. La totalité des massif se trouvent sur les feuilles Bouïra (66) et Tazmalt (67) de la carte topographique au $1 / 50000$.

\section{B. Aperçu géologique.}

Le Djurdjura fait partie de la dorsale kabyle (chaîne calcaire de L. Glangeaud), zone de hauts-fonds bordant au Mésozoïque et à l'Eocène le socle kabyle en partie émerge et proche de zones de flyschs dans lesquelles se sédimentaient d'épaisses séries terrigènes (Coutelle, 1976). Cette chaîne calcaire est complexe, formée de plusieurs unités (Coutelle, 1977). C'est à la description et l'interprétation de cet auteur que nous nous adressons ici. Notons que la cartographie détaillée du Djurdjura a d'abord été établie par Flandrin (1952). Dans ce très bref aperçu, nous ne retiendrons que les aspects qui nous intéressent directement.

Suivant l'unité à laquelle on s'adresse, la colonne stratigraphique est plus ou moins différente. En fait, seules deux d'entr'elles sont directement concernées par les principaux phénomènes karstiques décrits ici: l'unité du Ras timédouïne (Haïzer - Ras Timédouïne - Azérou-ou-Gougane - Azérou Thaltat) et celle de Tikjda (Ras Tigounatine - Terga Iroumienne - Adrar Timésouïne -Azérou Tidjer). Leur stratigraphie est très proche (Fig. 2a) et comprend de bas en haut:

- un Trias gréso-pélitique, puis dolomitique, et enfin à nouveau gréseux (avec dragées de quartz). Notons que l'unité de Tikjda possède en plus des couches 
plus anciennes (phyllades notamment).

- le Lias inférieur, assise fondamentale pour nous car constituée de calcaires massifs épais de $400 \mathrm{~m}$ au Ras Timédouïne.

- le Lias supérieur qui montre des calcaires et marno-calcaires en strates minces; ils jouent un rôle géomorphologique non négligeable grâce à leur aptitude à la gélifraction.

- des assises supérieures, plus marneuses (Albien à Sénonien) moins importantes pour nous.

Mentionnons encore des calcaires nummulitiques, trés abondants dans l'unité des Kouriet où ils sont karstifiés.

Les différentes phases tectoniques alpines ont bouleversé ces séries (Coutelle, 1976). Elles se caractérisent essentiellement par de très importants charriages du $\mathrm{N}$ au S qui ont provoqué le chevauchement sur une grande échelle des différentes unités: flyschs de différents types, socle kabyle, chaîne calcaire, matériel tellien (Fig.2b). De plus, la dorsale se retrouve elle-même sous la forme d'une série d'écailles correspondant aux différentes unités de la chaîne calcaire, empilées les unes sur les autres (Fig.2c). Le rôle de la néotectonique est primordial: elle a provoqué le soulèvement et la fracturation de ce dispositif, le redressant parfois presque à la verticale.

Cette histoire morpho-structurale a ainsi donné au Djurdjura ce cachet si caractéristique d'écailles successives possédant chacune une épaisse série calcaire qui se retrouve en position dominante. De là découlent ces hautes crêtes calcaires allongées parallèlement les unes aux autres et séparées par des dépressions subséquentes, dégagées dans des terrains plus tendres (détritiques du Carbonifère, Permo-Trias, etc...), ayant évolué soit en vallées ouvertes sur le bas pays par des gorges épigénétiques au travers des barres calcaires (Oued-elHammam par exemple), soit en cuvettes fermées avec écoulement souterrain (Tizi Boussouil, Quinif, 1977). Insistons encore sur l'intense tectonisation des massifs calcaires qui a une importance majeure sur les phénomènes karstiques.

\section{Eléments climatologiques.}

Peu de données sont exploitables. Les zones de haute altitude qui nous intéressent ne sont pas équipées. La carte pluviométrique (Chaumont et Paquin, 1971) indique que les zones situées à plus de $1700 \mathrm{~m}$ reçoivent plus de $2 \mathrm{~m}$ de précipitations annuelles. Ce sont ces régions qui recèlent la quasi-totalité des zones d'alimentation des réseaux karstiques. Aux environs de $1000 \mathrm{~m}$ d'altitude, la montagne reçoit encore entre 1200 et $1500 \mathrm{~mm}$ d'eau.

Autre donnée importante, une partie des précipitations se fait sous forme de neige. Aïn-el-Hammam (ex-Michelet), située à $10 \mathrm{~km}$ au $\mathrm{N}$ et à $1080 \mathrm{~m}$ d'altitude connaît 28 jours d'enneigement par an pour une pluviométrie de 1145 mm (Seltzer, 1946). 


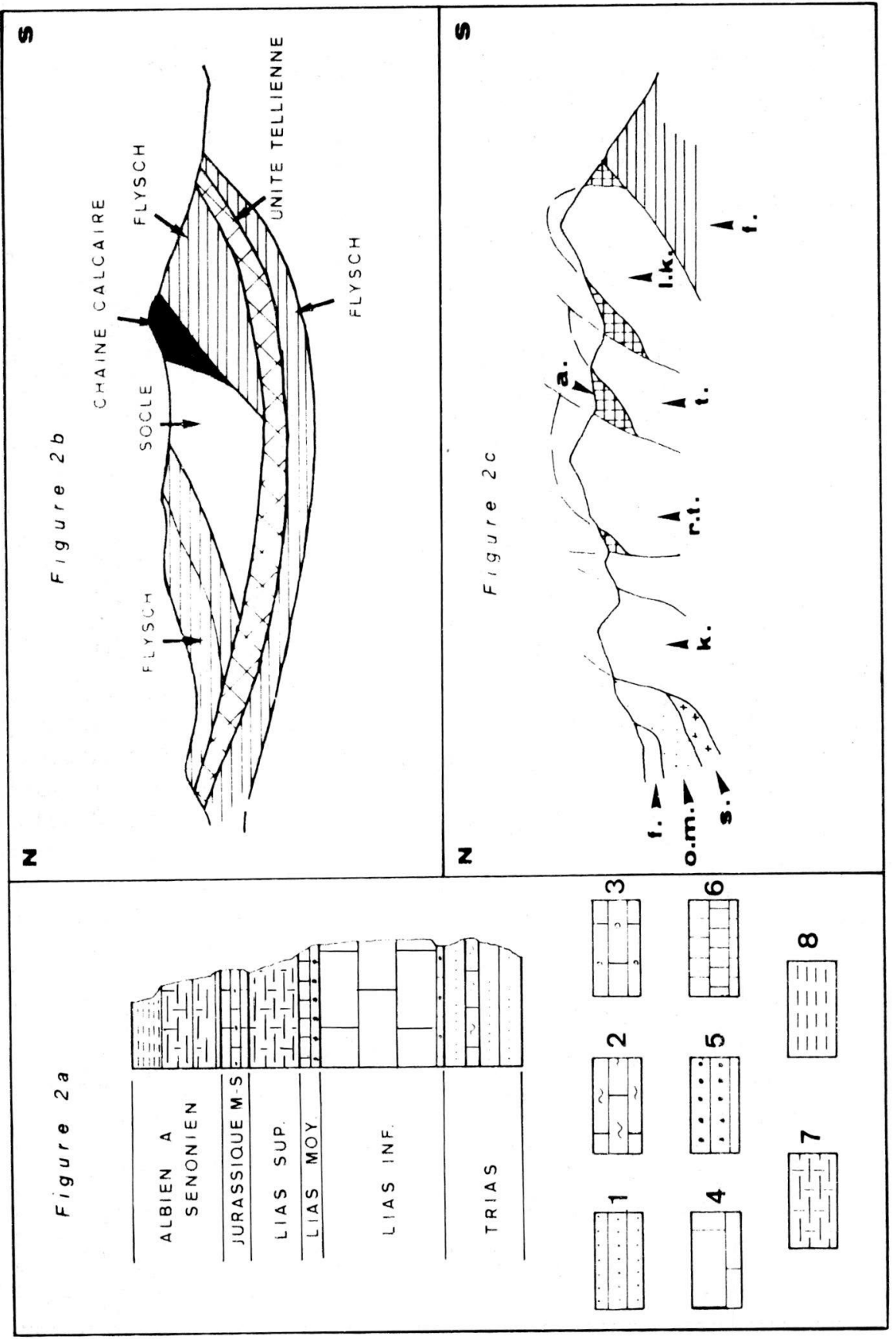




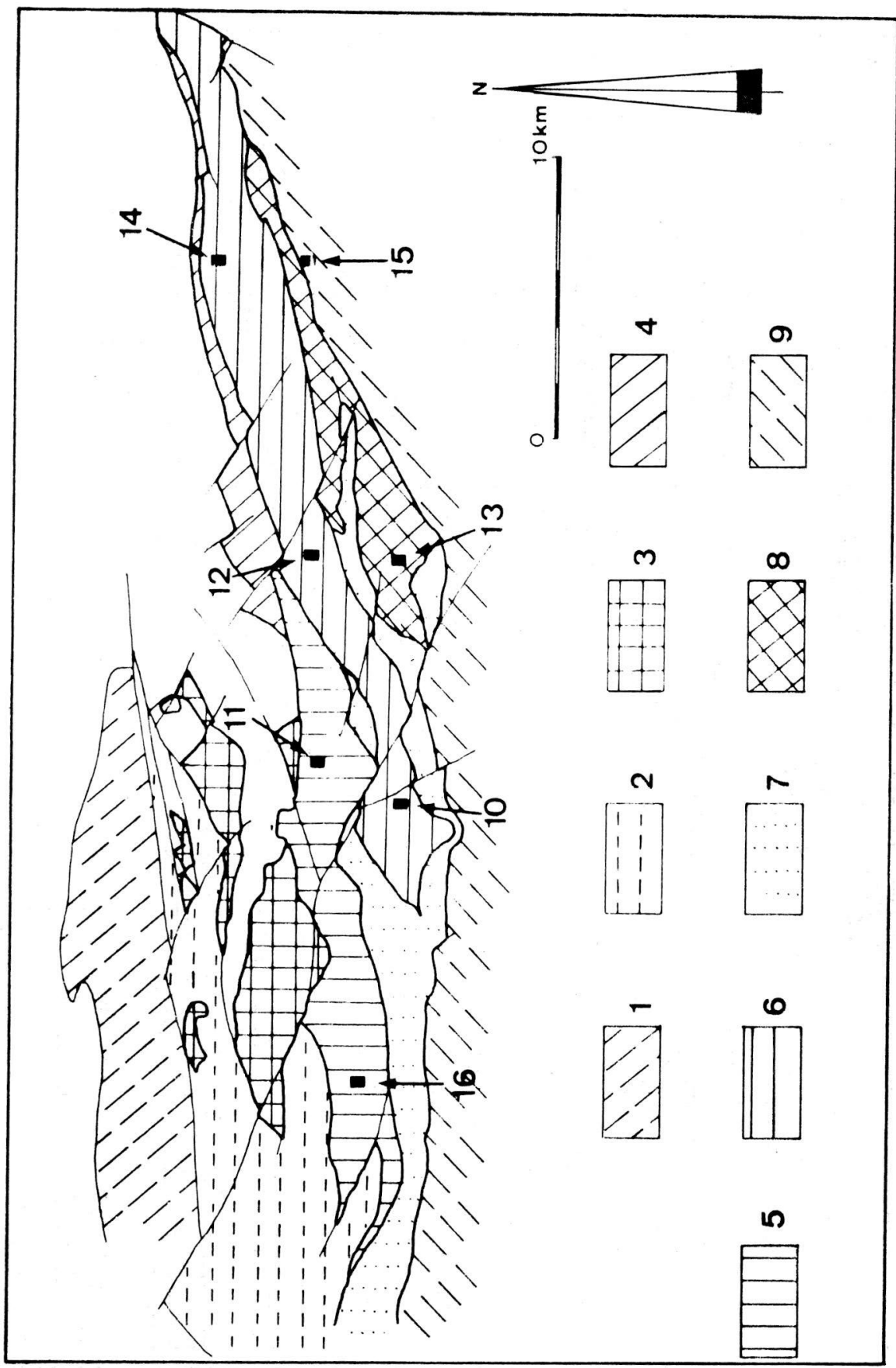


Fig.2a. Colonne stratigraphique de l'unité du Ras Timédouïne (d'après Coutelle, 1977, simplifié). 1. Grés.

2. Dolomie et calcaire dolomitique.

3. Calcaire oolitique.

4. Calcaire massif.

5. Conglomérat.

6. Calcaire fin rouge.

7. Marno-calcaire.

8. Marne.

Fig.2b. Coupe synthétique et hypothétique de la Grande Kabylie (d'après Coutelle, 1976, simplifié).

On constate que le Djurdjura (chaîne calcaire) est une unité parmi d'autres d'un vaste ensemble charrié. Les traits gras qui séparent les unités sont des contacts anormaux.

Fig.2c. Coupe transversale synthétique du Djurdjura (d'après Coutelle, 1977, simplifié).

Cette figure montre que le Djurdjura lui-même est constitué de diverses unités tectoniques qui se chevauchent l'une l'autre.

f.: flyschs; o.m.: Oligo-Miocène; s.: socle kabyle; k: unité des kouriet; r.t.: unité du Ras Timédouïne; t.: unité de Tikjda; a.: unité d’Adjiba; l.k: unité de Lala Khedidja. Sur ce schéma, la cuvette de tizi Boussouil se situerait approximativement en dessous de la lettre «a», entre les unités du Ras Timédouïne et de Tikjda. La cuvette S se creuse dans des formations tendres de l'unité d'Adjiba.

Fig.2d. Schéma structural du Djurdjura (d’après Coutelle, 1977, simplifié).

1. Flyschs.

2. Oligo-Miocène.

3. Unité des Kouriet.

4. Unité de l'Azrou Aïcha.

5. Unité du Ras Timédouïne.

6. Unité de Tikjda.

7. Unité d'Adjiba.

8. Unité de Lala Khedidja.

9. Flyschs du flanc sud du Djurdjura.

10. Tikjda.

11. Ras Timédouïne.

12. Tizi n'Kouilal.

13. Lala Khedidja.

14. Azérou Tidjer.

15. Col de Tirourda.

16. Djebel Haïzer.

Notons enfin que le Djurdjura connaît généralement plusieurs couvertures neigeuses par hiver . L'oscillation gel nocturne - dégel diurne est courante et favorise l'actuelle gélifraction.

\section{Les grands traits géomorphologiques.}

La première influence sur le relief du Djurdjura est d'abord structurale. L'érosion s'est exercée de façon différentielle sur des massifs soulevés par la néotectonique plio-quaternaire. Les couches continentales détritiques (conglamérats, argile) du flanc sud du Djurdjura attestent de cette érosion. De plus, la tectonisation de ces roches témoignent de mouvements simultanés et postérieurs à cette dégradation rexhistasique. 
Les axes des crêtes calcaires résistent à l'érosion plus que les autres assises et occupent ainsi une position plus que les autres assises et occupent ainsi une position dominante. D'autre part; les couches plus tendres évoluent en dépressions subséquentes servant de bassins de réception à des oueds importants. Creusées dans le Carbonifère, elle draine les torrents descendant des flancs calcaires. L'oued ainsi formé (oued-el-Hammam) sort épigénétiquement en tranchant la barrière calcaire Adrar Timésouïne - Azérou Tidjer. L'axe hydrographique Oued-el-Berd - Irhzer Tizi N'Kouilal contourne le Lala Khedidja en creusant les couches tendres du Carbonifère et du Paléogène.

\section{LE KARST SUPERFICIEL.}

Trois types de formes fondamentales se partagent la surface: les champs de lapiés, les dolines et les dépressions fermées de grandes dimensions (poljés). De plus, d'autres formes sont typiques du Djurdjura, la plus extraordinaire étant l'agouni.

\section{A Les champs de lapiés.}

Bien que d'étendue restreinte, ils donnent un cachet typique au karst djurdjurien.Ou bien les formes sont groupées en champs de lapiés, ou bien elles sont dispersées, entaillant d'autres formes (paroi de dolines, versant). Examinons rapidement les microformes fondamentales.

1) Les bogaz (karstgasse) sont souvent de très grandes dimensions. Ce sont des dépressions dont la largeur est de quelques mètres pour une longueur atteignant plusieurs dizaines de mètres et une profondeur comprise entre 10 et 20 mètres. Ils s'établissent au détriment de failles subverticales.

2) Les kluftkarren sont moins développés; ils ne sont pas typiques de ce karst.

3) Les formes de ruissellement peuvent être énormes. Les cannelures de mur atteignent 20 mètres de haut pour un diamètre de plus de $50 \mathrm{~cm}$. Elles entaillent en particulier les parois des bogaz où elles s'alignent parallèlement les unes ou autres. Les rinnenkarren et les maanderkarren se développent aussi très fortement sur de grandes dalles obliques.

4) Les aiguilles de lapiaz résultent de l'exacerbation de cannelures. Ces dernières, se développant et se recoupant, isolent des pitons aigus aux arêtes coupantes. Leur hauteur atteint souvent plusieurs mètres.

5) Des crypto-lapiés (se développant sous un remplissage) sont visibles au fond des grandes cuvettes de l'Haïzer. Ils sont actuellement en voie de dégagement. Ils se caractérisent par des formes douces, l'absence d'arêtes vives ou de mi- 
croformes de lapiaz aérien (cannelures, rinnenkarren, etc.).

Ces microformes se rencontrent parfois isolées (Haïzer, flanc $\mathrm{N}$ du Gougane): cannelures ou rinnenkarren sur le flanc des dalles. Dans le cas contraire, elles s'associent en champs de lapiés. Ces derniers montrent deux types opposés.

1) Les lapiés de fracturation (lapiaz de la Terga par exemple) se caractérisent par les grands bogaz et les nombreux avens-tesserefts (voir infra) ou associations cannelures-méandres. Ils se développent sur des surfaces dont la pente est inférieure à $45^{\circ}$.

2) Les lapiés de ruissellement établis là où la pente est forte, montrent surtout des rinnenkarren, des cannelures. Les petits lapiés du versant $\mathrm{S}$ du Gougane sont de ce type et se développent sur des versants très raides. On y compte moins d'avens, mais au contraire des couloirs cutanés qui s'étendent à faible profondeur sous le lapiaz.

Insistons sur le caractère haut-alpin de ces lapiés, l'exacerbation exceptionelle de certaines formes, telles les bogaz géants et les grandes cannelures.

\section{B. Les dolines (Fig.3).}

Leur distribution est irrégulière. La première chose à remarquer est leur tranche altimétrique qui s'étend de 1.500 à $2.200 \mathrm{~m}$, c'est-à-dire la même que les lapiés. Ici, à l'encontre de ce qui se passe pour les karsts haut-alpins européens, il n'y a pas distinction entre un étage à dolines suivi à plus haute altitude d'un étage à lapiés (Quinif, 1976a).

L'ensemble le plus remarquable est certainement le Tachgagalt, le «plateau aux dolines», dans la partie E de L'Haïzer. Les dolines y sont coalescentes et se comptent par dizaines sur un espace restreint. A certains endroits, elles ne sont séparées que par des crêtes aigües. Tous les types se rencontrent.

1) la doline-puits, au fond souvent occupé par un névé, a un rapport profondeur - largeur plus grand ou de l'ordre de I. Les parois sont verticales, entaillées par les cannelures de mur. Leur profondeur moyenne est de $15 \mathrm{~m}$, mais elles se prolongent parfois par un méandre et préfigurent alors un veritable aven.

2) La doline en cuvette est la forme opposée à la précédente. Elle est large et peu profonde. Les plus grandes, qui ont entre 50 et $100 \mathrm{~m}$ de diamètre, évoluent parfois vers la forme petit poljé avec plusieurs pertes localisées dans les flancs.

3) Les dolines en entonnoir constituent une forme intermédiaire, proches des dolines-puits, mais avec un rapport profondeur - largeur plus petit que I. Elles montrent aussi des cannelures de mur.

4) Les dolines en entonnoir assymétrique, formes parfois géantes (100 m de 




Fig. 3. Types de dolines (voir texte).

diamètre, $50 \mathrm{~m}$ de profondeur), sont nombreuses sur les versants. Elles ne contiennent pas de névé permaneǹt.

On rencontre également des dolines ailleurs sur le Djurdjura, mais en nombre beaucoup moins grand. Elles défoncent parfois les champs de lapiés (dolinespuits du champ de lapiaz de la Terga). On en trouve même sur les crêtes (dolines assymetriques de la crête de l'Azérou-ou-Gougane). Elles s'associent çà et là en dépressions plus complexes (Tachgagalt surtout).

D’une manière générale, les dolines ne permettent pas une pénétration au sein de la masse calcaire. A part quelques dolines-puits du Tachgagalt qui en constituent un stade élémentaire, elles ne se transforment pas en aven. Aucune cavité actuellement connue ne démarre par une doline.

\section{Les dépressions fermées de type poljé.}

Tizi Boussouil constitue l'archétype de la grande dépression fermée du Djurdjura, la plus connue aussi (Fig.4) Au fond d'une cuvette d'origine essentiellement structurale se rassemblent de nombreux talwegs qui fonctionnent en temps de crues (orages violents et fonte des neiges). L'exutoire de ces talwegs est le gouffre: l'Inker Temdat pour la cuvette N, l'Anou Boussouill pour la cuvette $S$.

Ce n'est pas un poljé au sens strict du terme: pas d'applanissement alluvial, 


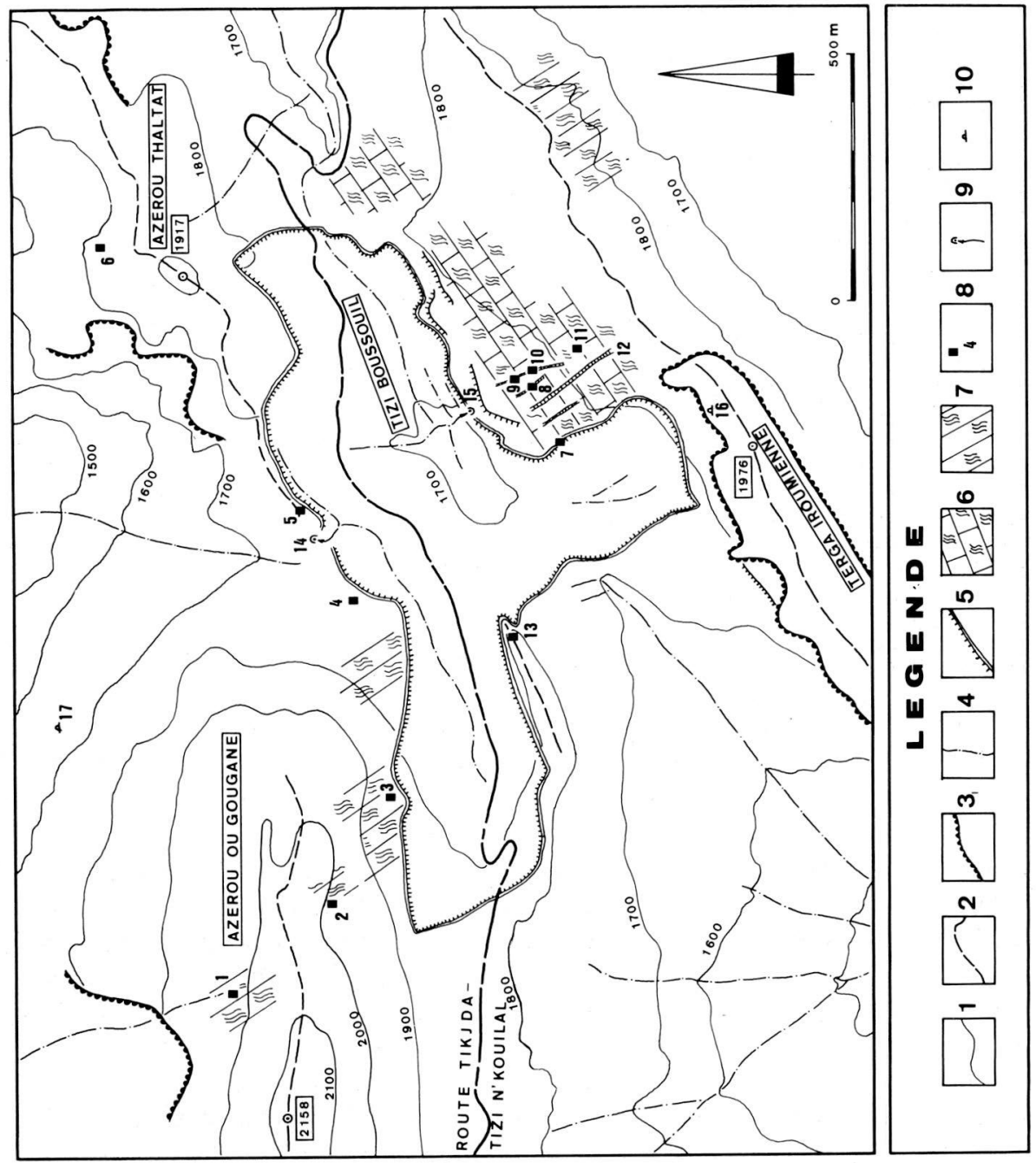


Fig.4. Région de Tizi Boussouill. (extrait de Quinif, 1977).

Légende:

1. Courbes de niveau (équidistance: $100 \mathrm{~m}$ ).

2. Axe des crêtes.

3. Parois verticales (plus de $50 \mathrm{~m}$.).

4. Axe de talweg.

5. Aven de $15 \mathrm{~m}$.

6. Lapiaz surtout de fracturation.

7. Lapiaz surtout de ruissellement.

8. Cavités (voir liste ci-dessous): avens.

9. Ponor.

10. Cavités: grottes de versants.

Liste des cavités: 1 . Tessereft de $30 \mathrm{~m}$ (P5-P20-P5).

2. Tessereft de $20 \mathrm{~m}$ (P15-P5).

3. Aven de $30 \mathrm{~m}$.

4. Groupe de 2 petits avens (P10).

5. Aven de $15 \mathrm{~m}$.

6. Aven de $25 \mathrm{~m}$ (P25).

7. Aven de $13 \mathrm{~m}$ (P13).

8. Tessereft Messaoud (plan infra).

9. Anou Manarf (-110: P15, méandre, P10, P20,P25).

10. Anou Pas-vu-pas-pris-(plan infra).

11 Aven de $30 \mathrm{~m}$.

12. Grand bogaz (section infra).

13. Aven de $15 \mathrm{~m}$.

14. Anou Inker Temdat (plan infra).

15. Anou Boussouill.

16. Grotte perchée de la Terga.

17. Anou Thinichiguine (plan infra).

pas d'engorgement des ponors, écoulement torrentiel. La cuvette doit sa position à la structure: situées sur les couches tendres et imperméables du Trias et du Paléogène, elle se trouve coïncée entre deux écailles calcaires (Lias inferieur). Ce sont donc les charriages qui, en faisant se chevaucher des écailles successives comprenant des assises calcaires et imperméables, est cause de cette disposition (Quinif, 1977).

Les poljés de l'Haïzer occupent une position structurale de même nature, sinon que les assises imperméables concernées sont différentes: bassin d'écoulement constitué par des conglomérats tertiaires, des marnes lutétiennes et sénoniennes. Les eaux s'écoulent ainsi vers le calcaire liasique où elles s'enfouissent sur le pourtour des bassins fermés. Ces derniers ont ici un caractère de poljé beaucoup plus marqué. Ils présentent des surfaces d'applanissement alluvial, les talwegs y sont beaucoup moins imprimés, les ponors s'engorgent lors des crues. Remarquons que la présence d'un crypto-lapiaz déjà cité montre que les cuvettes sont en voie de creusement par l'enlèvement de la couverture alluviale. La capacité des ponors augmente donc actuellement. 


\section{Les agounis.}

Il n'est pas possible de passer cette forme particulière sous silence. Se rapprochant d'un karstgasse, elle s'en distingue par ses caractères morphologiques et sa position topographique. Ce sont de grands couloirs en fond de bateau, montrant une direction préférentielle, profonds de plus de $50 \mathrm{~m}$ pour une largeur de quelques dizaines de mètres et une longueur supérieure à $100 \mathrm{~m}$. Leur fond est encombré d'éboulis gigantesques, renfermant des névés à ciel ouvert. Ils sont dûs à la conjonction entre une fracturaction tectonique très forte dont la direction principale est parallèle à un grand versant (agounis de la Dent du Lion sur l'Haïzer, agounis du Ras Timédouine).

\section{Les cavités.}

Compte tenu des moyens disposés durant les différentes expéditions, c'est surtout l'angle morphologique qui sera abordé. L'examen des dépôts n'a pu être que descriptif et superficiel. Quelques mesures chimiques ( $\mathrm{pH}$, dureté) ont été effectuées dans l'Inker Temdat et les tesserefts.

\section{A. Les gouffres-ponors (avens-chantoirs (I)).}

\section{1) Généralités - Description de l'Anou Inker Temdat.}

Ce sont des cavités à caractère vertical (présence de puits et de crans verticaux ou subverticaux se suivant en continuité ou reliés par des galeries à faible pente), absorbant massivement les eaux de ruissellement collectées au sein de cuvettes fermées de type polje. Pratiquement, seuls les avens de Tizi Boussouil permettent une pénétration apréciable dans la masse calcaire et, par là même, l'observation des formes, microformes et depôts caracteristiques: l'Anou Boussouil $(-505 \mathrm{~m})$ et l'Anou Inker Temdat $(-255 \mathrm{~m})$. Alors que le premier est relativement simple, le second montre une complexité plus grande et une richesse de formes intéressante. C'est lui que nous avons étudié plus particulièrement.

\section{L'ANOU INKER TEMDAT.}

a) Structure du gouffre et topographie. (Fig.5)

Le plan montre une topographie relativement compliquée. Essentiellement, le

(I) Chantoir est une dénomination d'origine belge qui a un sens morpho-hydrogéologique bien précis. Il s'agit d'une cavité à caractère vertical (donc un aven) qui engloutit ou a englouti un cours d'eau. Il se situe donc sur une rupture de pente importante dans le profil en long du torrent. Sa pente est plusieurs fois plus forte que la pente de la partie aérienne qui précède. Il constitue le chemin de l'eau entre la surface et la zone d'écoulement. Dans ce cas-ci, c'est un ponor, mais qui ne fait qu'absorber et cela par un système de puits. 
gouffre comprend trois parties: la partie «chantoir» de la cavité qui s'étend de l'entrée jusqu'au confluent en excluant le réseau Martine, ce dernier qui constitue une unité à part en temps qu'affluent du gouffre, et la rivière souterraine depuis le PI8 du réseau amont jusqu'à la voûte mouillante.

\section{b) Morphologie de la cavité.}

Les trois parties citées ci-dessus sont morphologiquement distinctes. Examinons-les successivement.

\section{1) L'aven chantoir.}

La fonction hydrogéologique est nette: le gouffre absorbe un torrent lors des crues. C'est donc bien un chantoir tout-à-fait typique présentant une pente importante ( $40 \%$ en moyenne jusqu'au confluent).

La forme principale est le puits. Les P35, P40, P27 constituent des drains verticaux amenant rapidement les eaux en profondeur. Nous avons donc ici la partie supérieure de la zone d'alimentation d'un réseau perte résurgence. Les passages plus horizontaux ne sont pas des collecteurs: c'est une succession de ressauts séparant deux puits. Cet ensemble correspond à la première classe de puits considérée par Renault (1968) dans laquelle ces associations puitsgaleries forment un ensemble très incliné typique des karsts de montagne (régime de détente mécanique généralisée, voir plus loin).

La morphologie de ces puits est particulière. Ils se développent au détriment de joints de stratification très redressés $\left(75^{\circ}\right)$, ce qui explique plusieurs caractères. La voûte est constituée par la surface inférieure de la strate à peine retouchée. L'inclinaison de la partie supérieure du puits est due au pendage. La partie inférieure s'en affranchit grâce au creusement plus accentué et tend vers une forme en éteignoir (Fig.6). On peut donc voir là une évolution entre une fente peu touchée et le vaste puits entièrement en éteignoir.

\section{2) Le réseau Martine.}

Nous ne sommes plus en présence de puits mais d'une galerie conduisant vers l'amont à un élargissement important (à -70). Ce réseau ne constitue plus une cavité absorbante du genre aven mais une portion de zone d'écoulement venant se greffer sur le chantoir. La salle, dont le sommet se perd dans le noir, fait d'ailleurs songer au bas d'un puits et constituerait alors la partie inférieure de la zone d'alimentation d'un petit réseau affluent.

\section{3) La rivière.}

La rue d'eau présente également un caractère différent de l'aven-chantoir. La pente moyenne, bien que restant forte $\left(30^{\circ}\right)$, est plus faible que celle du chantoir. Nous ne trouvons plus de drain profond; les changements brusques de ni- 


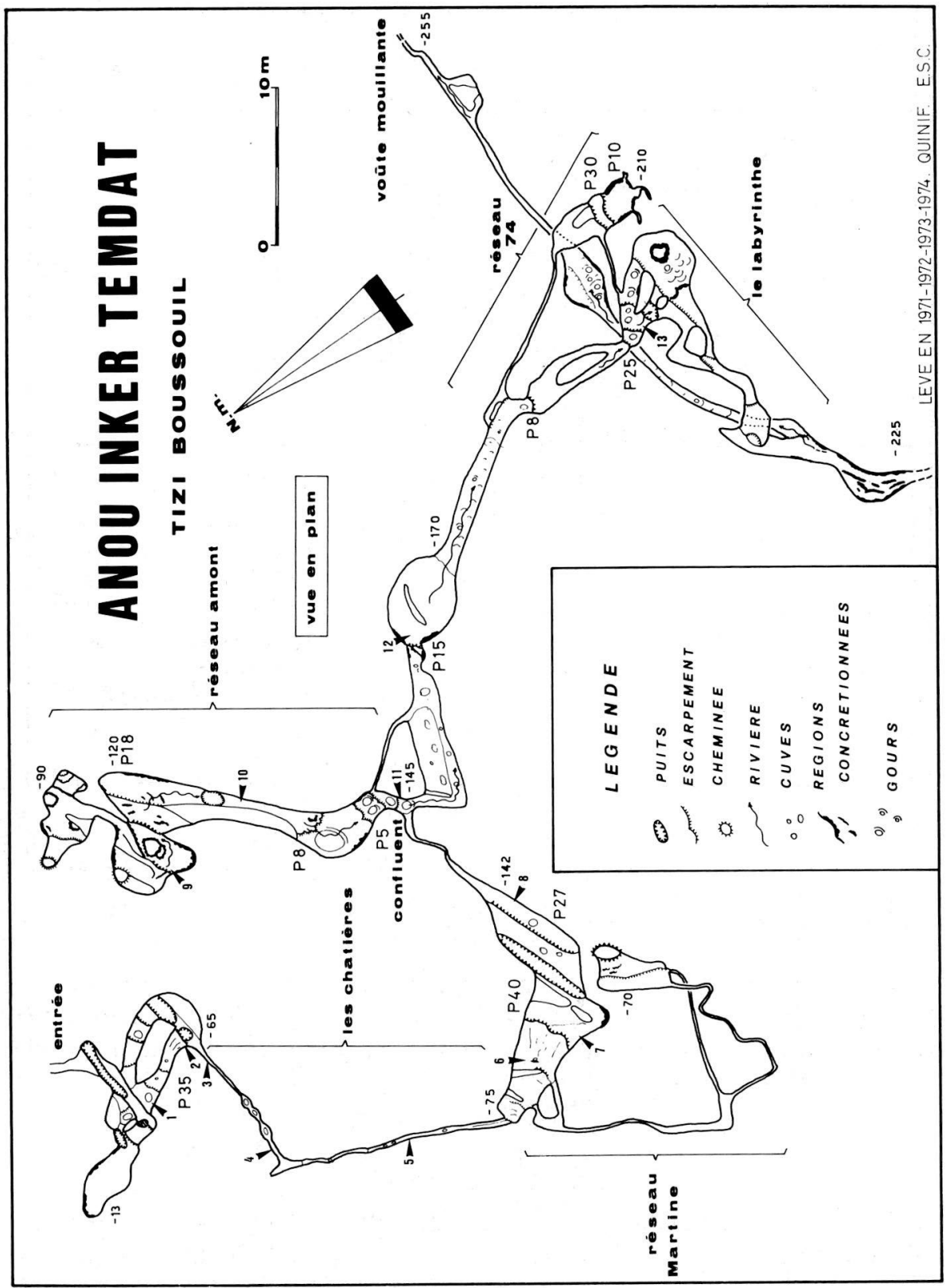






Fig.5 . Anou Inker Temdat: plan et coupe.

Les numéros indiquent les endroits des prélèvements d'eau (tableau $n^{\circ} 1$ ).

veau de la rivière (5 puits arrosés principaux) ne constituent plus des engouffrements profonds mais des crans de descente dans une galerie. Les formes et concrétions (voir plus loin) sont d'ailleurs peu caractéristiques de la forme puits au sens strict du terme.

c) Microformes et dépôts.

\section{1) Dans l'aven-chantoir.}

Les cuves sont très nombreuses, à chaque palier, dans les chatières et même au milieu des puits obliques. Elles sont de toutes les tailles, depuis la dizaine de 


\section{STRUCTURE DE PUITS}

\section{INKER TEMDAT}

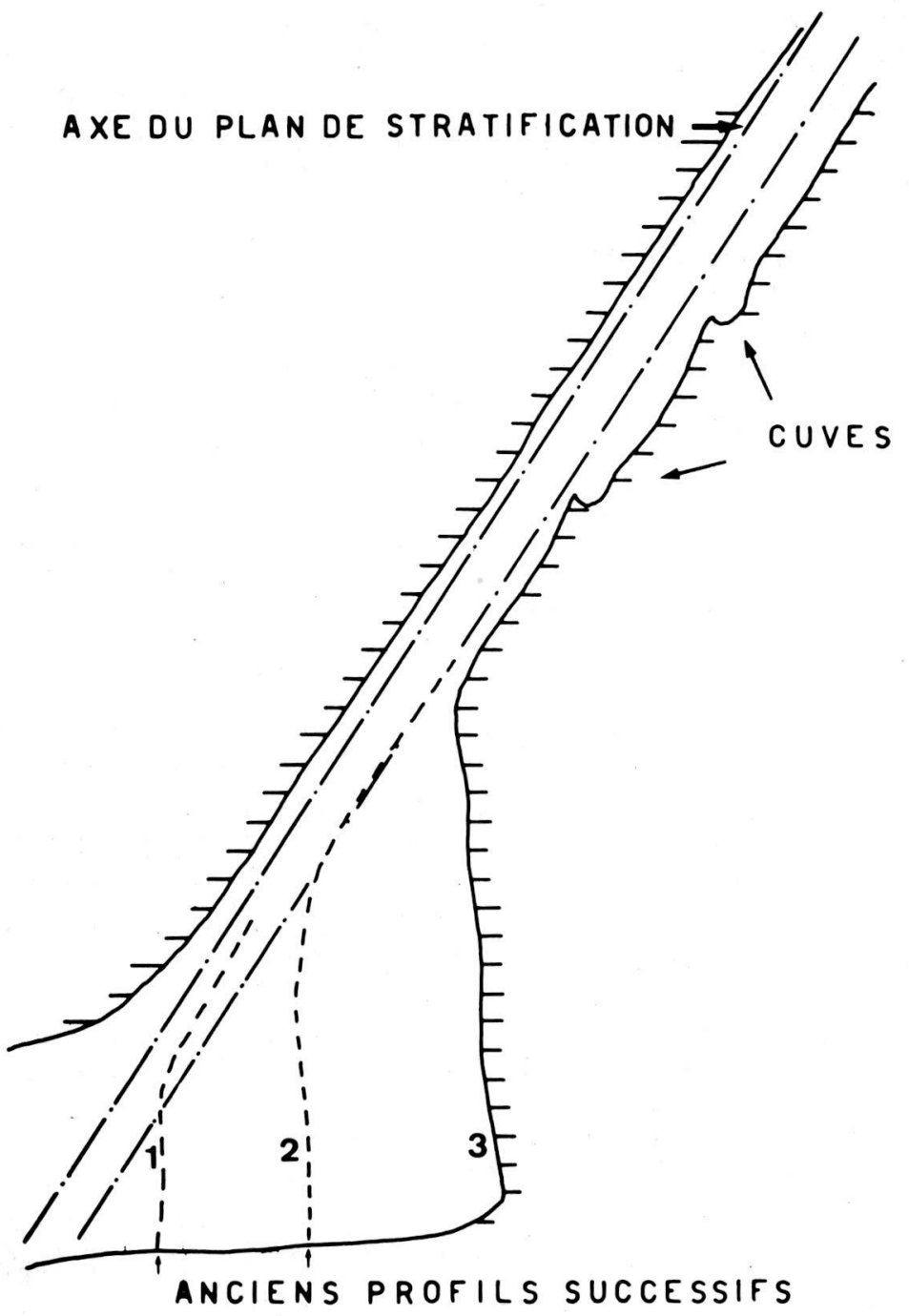

Fig. 6a. Ce schéma de principe montre une coupe au travers d'un puits-type de l'Inker Temdat. Ce puits se développe suivant deux plans de stratification. On a indiqué les profils successifs d'écoulement. L'eau suit d'abord les plans de stratification pour s'en affranchir plus tard en écoulement cascadant avec formation de cuves. Le bas du puits prend un profil en éteignoir, ce dernier remontant progressivement vers le sommit du puits. Dans cette évolution, la voûte est à peine touchée par l'érosion. 


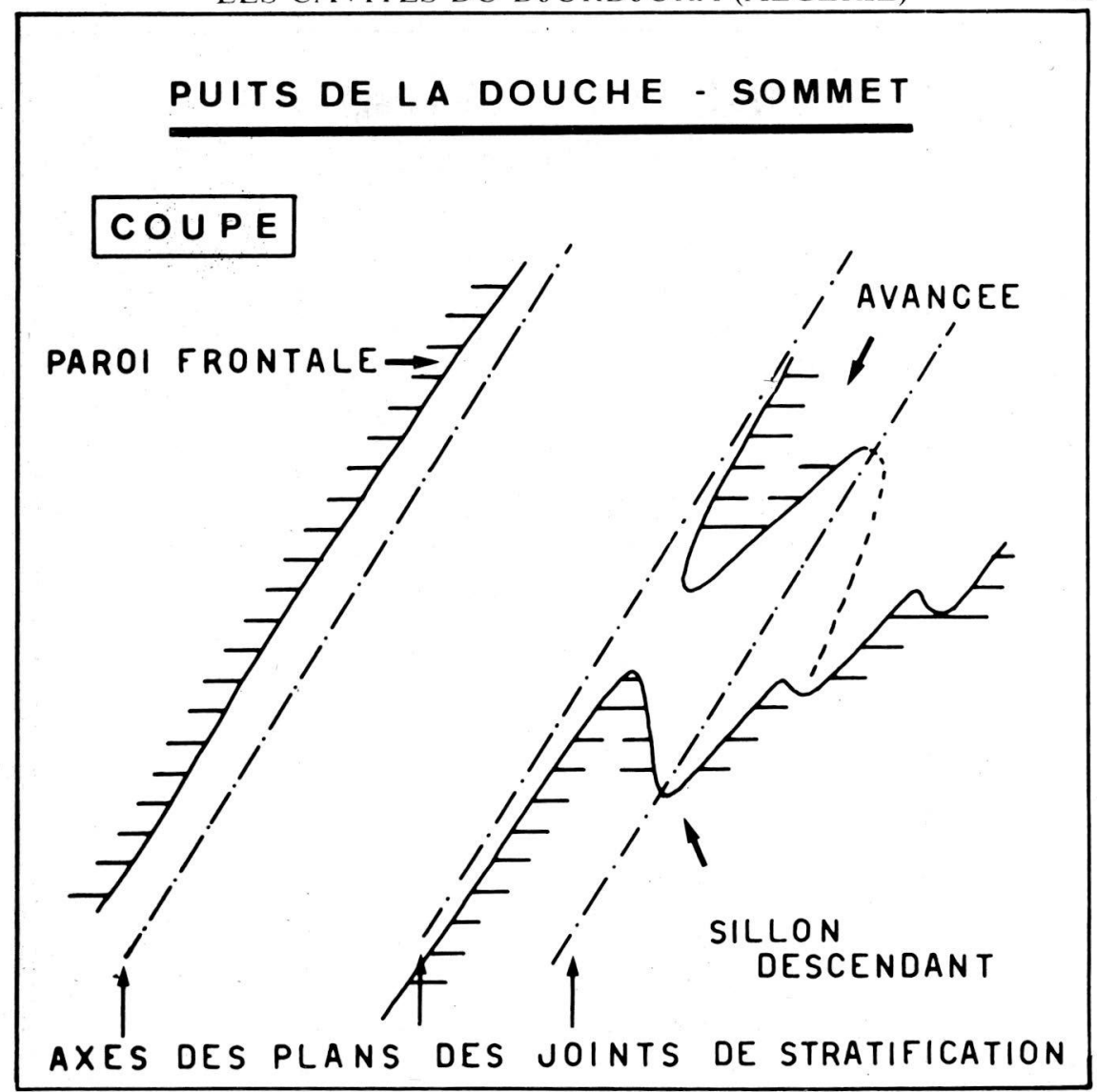

Fig. 6b. Cette coupe illustre le schéma de principe de la figure 6 a quant au contrôle tectonique sur la morphologie d'un puits particulier: le P40 (puits de la «douche»).

centimetrès jusqu'à plus de $2 \mathrm{~m}$. La profondeur dépasdse parfois le mètre. Dans certaines marmites de géants, on trouve de magnifiques meules de grés ou de calcaire.

Les parois lisses se rencontrent là où le courant est violent et où des ruissellements permanents corrosifs sont absents.

Les cupules et dentelles de pierre dépendent de ces ruissellements. Elles se développent aux endroits protégés de l'action du torrent.

Les cannelures caractérisent le P40 surtout. Elles entaillent la partie oblique supérieure. Elles présentent des analogies avec les cannelures de lapiés, mais elles sont moins rectilignes et moins régulières.

Des surcreusements se voient dans les chatières et la galerie précédant le confluent. 
Les sédiments sont peu abondants dans ces conduits balayés par les crues. Les fins se rencontrent dans de petites zones de décantation (petit galets, sable grossier). En plusieurs endroits, on rencontre les traces d'un ancien niveau visuaslisé par des débris de plancher stalagmitique cimentant des galets roulés (à $1 \mathrm{~m} \mathrm{du}$ fond du P35 par exemple). Les concrétions stalagmitiques sont rares. Quelques coulées s'oservent dans le bas du P35. Avant le P40, un surcreusement entaille un plancher stalagmitique stratifié ainsi que le bed-rock inférieur (Fig.7).

\section{COUPE DANS UNE CHATIERE}

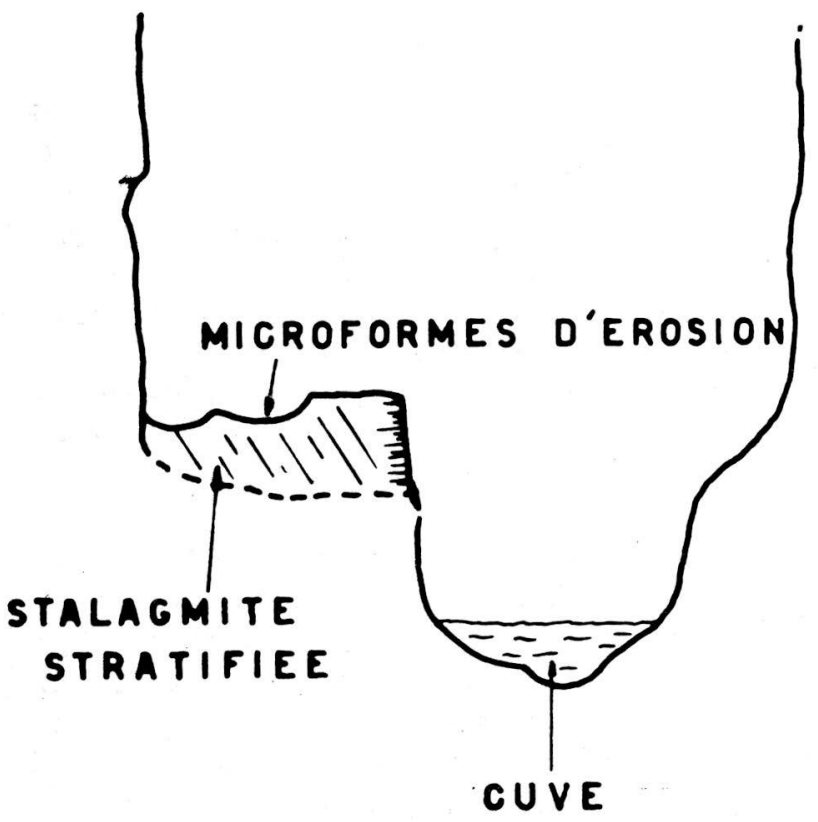

Fig. 7. Cette section au débouché de l'ensemble de chatières entre le P35 et le P40 de l'Inker Temdat montre le ruisseau actuel coulant dans un surcreusement entaillant à la fois un ancien dépôt stalagmitique montrant des couches successives et le bed-rock.

De plus, ce dépôt stalagmitique présente à son sommet des microformes (petit cuves, cannelures). 


\section{2) Dans le réseau Martine.}

Aucune forme d'érosion ne se remarque. Par contre, une intense corrosion se manifeste par des formes déchiquetées. La galerie est dépourvue de sédiment grossier, seuls des limons ou de l'argile y sont présents. Ces indices infirment un courant violent.

\section{3) Dans la rivière.}

Le lit de la rivière est parsemé de gours, de coulées stalagmitiques. Les puits arrosés s'ornent de bourrelets et coulées stalagmitiques sur le passage de l'eau. Ces concrétions ne présentent pas de trace de corrosion (cannelures par exemple). Par contre, les parois des galeries sont très déchiquetées. On rencontre à nouveau des cuves, mais moins nombreuses que dans la partie chantoir. Très peu de sédiments détritiques sont présent. Seule la partie inférieure du gouffre présente des parois recouvertes d'argile gluante marquant la zone de décantation en amont de la voûte mouillante.

\section{d) Chimisme des eaux.}

Les galeries de la rivière montrent deux influences opposées: une intense corrosion ayant généré des formes déchiquetées sur les parois et un concrétionnement dans le lit même de la rivière. Dans le partie chantoir, il y a les traces d'un ancien concrétionnement surcreusé. Pour tenter d'approcher le problème avec quelques données quantitatives, le $\mathrm{pH}$, la température et la dureté totale ont été mesurés en divers points du gouffre. Les résultats sont consignés dans le tableau I. Trois remarques préliminaires sont à faire:

- La température de l'eau dans le chantoir est assez constante, aux environs de $5{ }^{\circ} \mathrm{C}$; la température de la rivière est plus élevée et diminue avec la profondeur. $\mathrm{L}^{\prime}$ 'eau débouche dans le gouffre à $6.5^{\circ} \mathrm{C}$; la température se stabilise plus bas à $5.7^{\circ} \mathrm{C}$.

- Le $\mathrm{pH}$ est constant. On peut distinguer une légère décroissance dans la rivière avec la profondeur.

- Les variations de la dureté sont significatives. Dans la chantoir, elle passe de $155 \mathrm{mg} / \mathrm{l}$ pour la valeur minimale à $175 \mathrm{mg} / \mathrm{l}$ pour la valeur maximale (en équivalents $\mathrm{CaCO}_{3}$ ). L'erreur relative est de $5 \%$. Ces variations ne semblent pas systématiques. Par contre, dans la rivière, la dureté est de $250 \mathrm{mg} / \mathrm{l}$ à l'entrée dans le gouffre et de $193 \mathrm{mg} / \mathrm{l}$ au bas du P25.

Cette diminution de la dureté avec la profondeur corrobore les observations, à savoir un concrétionnement abondant dans le lit de la rivière. La diminution de la température ne permet pas d'expliquer cette précipitation par un réchauffement de l'eau. Si nous pouvons admettre un réchauffement à l'entrée de l'eau dans le gouffre, comment le concrétionnement pourrait-il se produire plus bas alors que la température augmente? Il faudrait faire l'hypothèse que 
le réchauffement initial a été suffisant pour que le déséquilibre ainsi créé subsiste malgré le réchauffement ultérieur. Néanmoins, ce réchauffement est faible et le concrétionnement reste abondant jusque la voûte mouillante.

Une autre explication est de supposer que la rivière provient d'un milieu à concentration de $\mathrm{CO}_{2}$ plus important que dans le gouffre (fissures par exemple). Le passage dans le gouffre provoque alors une libération de $\mathrm{CO}_{2}$ avec précipitation de carbonate.

Les caractères de l'eau à son entrée dans le gouffre donnent matière à réflexion sur son origine. 4 possibilités s'offrent à nous: la fonte de névés souterrains, la vidange d'un réseau de fentes, la condensation souterraine, l'eau d'imbibition de la couche d'altérites sur les grés et marnes. Sa température relativement élevée semble exclure une origine nivale. Compte tenu de petites sources qui proviennent de la couche d'altérites, il semble plausible qu'une partie au moins de la rivière ait cette origine.

\section{Tableau n. 1: résultats des mesures chimiques}

\begin{tabular}{cccc}
\hline Station & $\begin{array}{c}\text { Témperature } \\
\left( \pm 0.2^{\circ}\right)\left(\mathrm{en}^{\circ} \mathrm{C}\right)\end{array}$ & $\mathrm{pH}( \pm 0.2)$ & $\begin{array}{c}\text { Concentration de } \\
\text { CaCO3 (en mg/l) } \\
\text { (erreur relative: } 5 \%)\end{array}$ \\
\hline 1 & 5 & 6,7 & 167 \\
2 & 5 & 6,7 & 155 \\
3 & 5 & 6,7 & 155 \\
4 & 5 & 6,8 & 175 \\
5 & 5,4 & 6,6 & 172 \\
6 & 5,2 & 6,7 & 170 \\
7 & 5,3 & 6,5 & 156 \\
8 & 5,3 & 6,5 & 156 \\
9 & 6,5 & 6,9 & 250 \\
10 & 6,4 & 6,8 & 217 \\
11 & 5,7 & 6,7 & 213 \\
12 & 5,7 & 6,6 & 193 \\
13 & 5,7 & 6,6 & 200 \\
\hline
\end{tabular}

Les emplacements des stations sont indiqués sur le plan et la coupe de l'Inker Temdat par les numéros correspondants. Les stations 1 à 8 se situent dans le chantoir, les stations 9 à 13 dans le cours de la rivière.

Quant aux microformes de corrosion sur les parois de la rue d'eau, elles s'opposent à l'actuel chimisme de la rivière qui concrétionne. Nous pouvons invoquer le mécanisme explique par Trombe (1952) consistant en une évaportation des eaux de la rivière, condensation au contact des parois avec corrosion et retour à la rivière. Néanmoins, les microformes résultantes ne montreraient pas une telle abondance d'anneaux, dentelles, becquets (certains dépassent le mètre de long pour un pédoncule épais de $10 \mathrm{~cm}$ environ). Sans nier cette action, cette corrosion doit être imputable à la rivière ce qui nous oblique à admettre une modification radicale de son chimisme au cours du temps. 
Une modification analogue se remarque dans le chantoir. Nous y distinguons au moins trois phases: une première érosive, une seconde montrant un concrétionnement et une troisième à nouveau érosive ayant démantelé le concrétionnement précédant. La tablette de galets cimentés au bas du P35 prouve que, à la fin de la première phase, un torrent circulait déja dans le gouffre, avec façonnement et transport de galets. L'Inker Temdat jouait donc déjà le rôle d'aven-chantoir. La modification du chimisme et de l'hydrogéologie a une provenance climatique. Actuellement, l'analyse nous montre les eaux des cuves et des petits écoulements dans le chantoir très aggressive. Elle continue à connaître une phase concrétionnante (avec érosion mécanique également, corroborée par la présence de cuves en aval du confluent, cuves absentes à l'amont).

Pour conclure, nous distinguons dans la partie chantoir trois phases. Actuelle-, ment, un action érosive importante se caractérise par les cuves avec meules, les galets, les parois lisses tandis qu'une corrosion est prépondérante à l'étiage. L'abrasion s'explique par un important débit solide. La corrosion est due à de petits ruisselets (réseau Martine, chatières) et probablement aussi à de l'eau de condensation. Cette action érosive a surcreusé des couches stalagmitiques et des lits de galets cimentés par la calcite témoignant d'une (ou de plusieurs?) phase concrétionnante précédente. Cette dernière a elle même suivi une autre phase à dominante érosive. On ne retrouve pas trace d'autres phases. La rivière ne montre pas de phase actuelle érosive (sauf des effets abrasifs ). Les microformes trahissent une phase corrosive plus ancienne. Il est tentant de mettre en parallèle les phases de la rivière et du chantoir. Les causes de ces changements sont probablement climatiques, bien que les différences actuellement observées incitent à la prudence.

\section{2) Les avens-chantoirs dans le contexte morpho-structural.}

La cuvette de Tizi Boussouïl est complexe, centrée sur des assises imperméables plus tendres que les barres calcaires environnantes et ayant évolué en dépression. A cause de sa position topographique (perchée à plus de $1000 \mathrm{~m}$ au dessus des niveaux de base), ses pertes sont des avens-chantoirs présentant de fortes pentes et constituant une rupture de pente. L'ampleur des gouffres de Tizi Boussouïl s'explique en partie par l'abondance des eaux perdues. Les torrents drainent un bassin assez vaste et, surtout, la fonte des neiges fournit en un temps court un grand volume d'eau, accompagné d'un fort débit solide. L'oued principal alimentant l'Inker coule sur des grés triasiques, ceux qui se perdent dans l'Anou Boussouïl ont imprimé leurs talwegs dans les marnes. Nous sommes donc en présence de deux réseaux du type perte-résurgence allochtone. Les avens sont des pertes de torrents temporaires d'où présence de cuves, galets, lessivage des sédiments fins, etc... 
La comparaison entre les deux abîmes est intéressante. Leur localisation dans le relief est différente. Alors que l'Anou Boussouïl constitue un drain vertical au milieu d'une imposante barrière calcaire (premiers contreforts de la Terga Iroumienne), l'Inker Temdat ne s'imprime pas dans le paysage: son entrée se trouve presqu'à la même altitude que le petit col le séparant de son torrentexutoire aérien. Cette différence tient peut-être en partie dans l'âge différent des deux cavités: L'Anou Boussouill, plus vieux, s'imprime de façon plus caractéristique dans le paysage. D'autre part, l'énergie hydrodynamique mise en oeuvre dans les bassins versants est différente, beaucoup plus importante pour l'Anou Boussouïl. La tectonique joue aussi un rôle; le grand gouffre doit sa position à une zone très tectonisée de la Terga, et notamment à une grande faille au détriment de laquelle la moitié inférieure du gouffre est creusée (Birebent, 1948). Les autres conduits empruntent des joints de stratification redressés à la verticale et quelques diaclases. Ces fractures sont très visibles dans le lapiaz surmontant le gouffre. Cela confère au plan de l'Anou une relative simplicité. Les eaux gagnent rapidement de la profondeur par une voie toute tracée. En d'autres termes, on peut dire que l'énergie mécanique dissipée dans les roches est tellement intense qu'elle a permis une concentration très forte d'énergie hydrodynamique qui a généré de grands conduits.

Cette simplicité ne se retrouve pas dans le plan de l'Inker Temdat qui montre peu de directions privilégiées. Cet enfouissement ne profite ici ni de faille, ni de diaclase importante. Il existe donc d'autres conditions favorables, résidant dans l'aspect mécanique du problème.

Depuis Renault (1967), l'importance de la mécanique des massifs est apparue comme un facteur fondamental intervenant dans la karstification. Dans le cas du Djurdjura tel qu'il se présente sous sa forme actuelle, nous avons affaire à un karst de montagne sous une forme très pure. Tout le massif est soumis à un régime de détente mécanique due à la fois à sa position dominante (versants de plus de $1000 \mathrm{~m}$ de dénivelée pour une largeur faible) et aux grands mouvements néotectoniques en extension. Lorsque de grandes fractures existent, elles sont naturellement exploitées par la karstification (Anou Boussouïl). Mais en leur absence, toutes les autres fractures de moindre importance, grâce à ce régime de détente, ont aussi la possibilité d'être exploitées. Le plan d'une cavité se développant au détriment de ces dernières sera donc plus compliqué, obéissant aux multiples sollicitations géométriques. C'est le cas de l'Inker Temdat. De toute façon, la résultante, dans un cas ou dans un autre est une cavité dans laquelle puits et galeries se suivent en continuité avec une pente très forte dans la zone d'alimentation (la seule connue à ce jour par les explorations). Notons que les puits de l'Inker Temdat sont creusés dans des joints de stratification parallèles au versant, autre manifestation de la détente due à la topographie. 


\section{3) Aspects hydrogéologiques.}

Les deux gouffres de Tizi Boussouïl constituent une partie de la zone d'alimentation de deux réseaux de type perte-résurgence. Les eaux courantes se réunissent dans les cuvettes et s'échappent par les abîmes.

Jusqu'ici, l'exploration de l'Anou Boussouïl n'a montré que la zone d'alimentation. La forme principale est le puits, la galerie en temps que collecteur n'étant pas représentée. Par contre, l'exploration de l'Inker Temdat a apporté d'autres éléments. La présence du Réseau Martine et de la rivière montre que d'autres unités se greffent sur le réseau de type perte-résurgence représenté par le chantoir. Ce sont probablement des réseaux dont la zone d'alimentation est située sous les lapiés voisins. L'Anou Boussouïl présente également des exemples de confluence. D'autre part, les avens du lapiaz de la Terga (voir plus loin) permettent d'explorer une partie d'une zone d'alimentation qui procède des infiltrations, de la fonte des névés souterrains et de la neige accumulée dans le lapiaz. Ces eaux confluent dans le massif avec les eaux perdues dans le ponor. Ce dispositif montre clairement les deux influences hydrogéologiques qui gouvernent ce réseau. Nous reviendrons dans la synthèse sur cet importante question.

\section{B. Les avens de lapiaz.}

\section{1) Généralités.}

Ce sont des cavités verticales s'ouvrant dans les lapiés, sur les versants, au creux des dolines. Elles constituent des cavités d'absorption situées à l'écart des pertes massives. Elles collectent les infiltrations, les eaux de fonte des névés souterrains et de la neige s'accumulant dans les lapiés. Plusieurs types d'avens sont à distinguer.

\section{2) Les avens-tesserefts.}

Typiquement, il s'agit d'une cheminée ou d'un puits suivant qu'on l'aborde par la base (passage latéral) ou par le sommet (Fig.8). Le fond est constitué d'un agglomérat de pierres surmontées de terres noires, le tout supportant un névé d'importance variable. Les dimensions du puits sont la plupart du temps modestes, n'excédant pas une dizaine de mètres au niveau du névé. La profondeur est variable, souvent comprise entre 10 et $50 \mathrm{~m}$. Les névés sont souvent permanent. Ils présentent néanmoins une importante fusion en été. La température de l'air, au niveau du névé, fluctue autour de $1^{\circ} \mathrm{C}$.

Le Tessereft Messaoud (Fig.9) est un tessereft important. Il s'ouvre dans le lapiaz de la Terga Iroumienne, au creux d'un bogaz. Creusé au détriment d'une faille subverticale, il plonge de $100 \mathrm{~m}$ pratiquement d'un seul jet. A - $30 \mathrm{~m}$ se trouve un premier névé (qui disparaît vers la fin-août) posé sur un palier formé 


\section{STRUCTURE D'UN TESSEREFT}

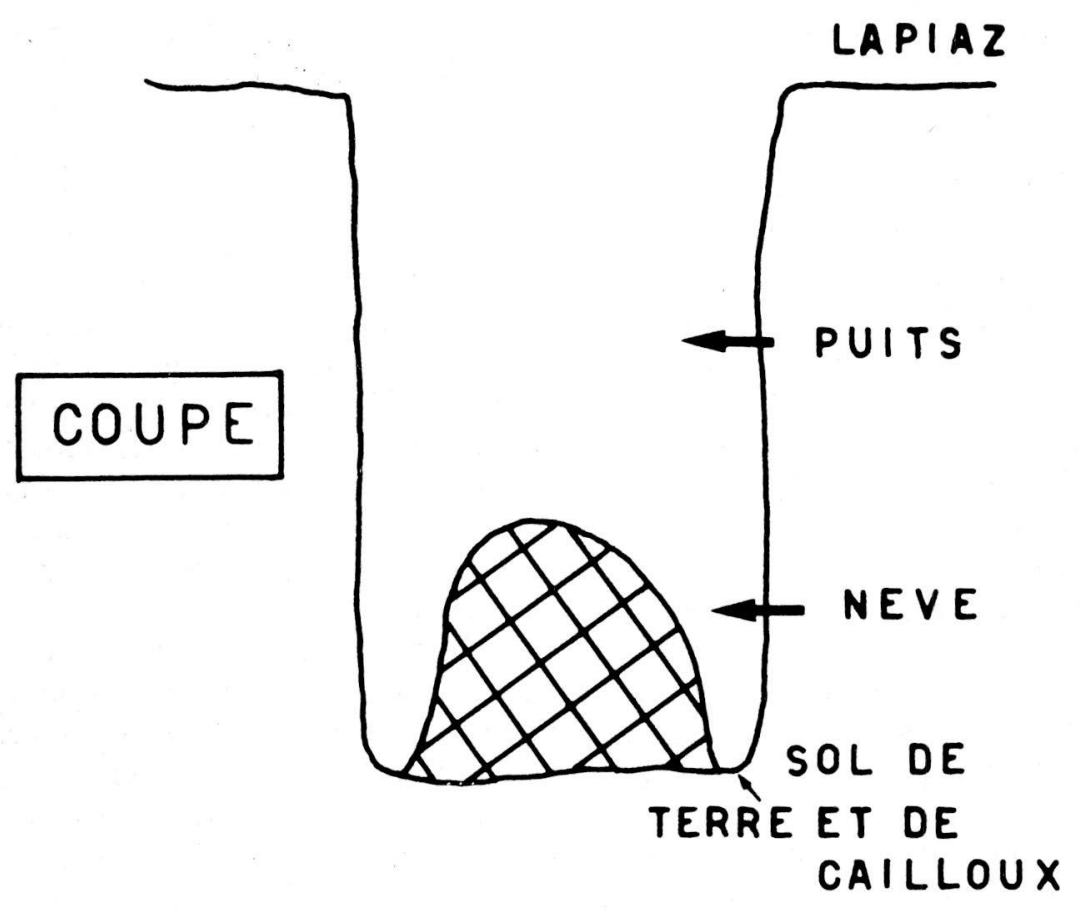

PLAN

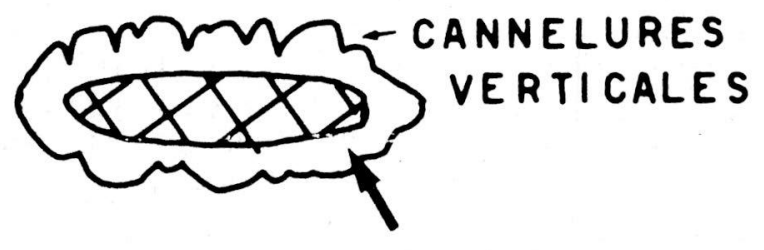

NE VE

Fig. 8. Structure-type d'un aven-tessereft simple. 
de gros blocs coïncés dans le grand puits. On le franchit latéralement. Notons que ce dispositif doit être celui de la plupart des tesserefts où le bouchon est impénétrable. Le second névé s'étend verticalement sur plus de 15 mètres (un puits de $20 \mathrm{~m}$ permet d'en parcourir une partie).

Remarquons qu'à - $80 \mathrm{~m}$, une petite faille perpendiculaire à la première et montrant une brèche de broyage donne à l'aven son ampleur. Du lapiaz jusqu'à -30 m, d'énormes cannelures découpent la paroi normale. Elles atteignent plus de $50 \mathrm{~cm}$ de diamètre. Plus bas, les parois deviennent surplombantes (P40).

Il est incontestable que les eaux de fonte des névés sont corrosives et génèrent ces microformes. A la base du premier névé, l'eau de fonte, non encore chargée, a donné un $\mathrm{pH}$ de 5 .

\section{3) Les systèmes cannelures-méandres.}

C'est dans le lapiaz de la Terga que nous trouvons les exemples les plus frappant de ce type de cavité d'absorption. Des failles transverses aux écailles de calcaire sont transformées en bogaz, ces derniers présentant une coupe assymétrique du fait du pendage de la faille $\left(60\right.$ à $\left.70^{\circ}\right)$. La paroi surplombante est à peine touchée par la corrosion tandis que l'autre est découpée par de gigantesques cannelures déjà décrites. Plusieurs cannelures peuvent confluer à leur base et atteindre ainsi jusqu'à $1 \mathrm{~m}$ de large et plus (Fig.10).

Ces grandes cannelures se prolongent vers le bas par des conduits en méandre quasiment verticaux, aux parois lisses, entrecoupés de murs de cascade. Les plus importants sont situés sur de petites failles perpendiculaires à la faille directrice. Ces galeries en trouvent partout à la limite du coïncement.

Le «grand bogaz» (Fig.4) compte 16 départs explorables jusqu'à $40 \mathrm{~m}$ de profondeur. Deux autres avens, situés dans un bogaz voisin du tessereft Messaoud, sont plus profonds. L'Anou Pas-vu-pas-pris (Fig.11) permet une pénétration à $-160 \mathrm{~m}$, plusieurs méandres (dont l'un présente un puits de $55 \mathrm{~m}$ ) confluent en un méandre collecteur. Cet aven constitue donc un stade plus avancé du dispositif où commence une certaine intégration. A de nombreux endroits de la cavité, on retrouve à la voûte la paroi surplombante de la faille, à peine touchée par la corrosion. Des failles perpendiculaires, à zone de broyage, sont aussi visibles. Comme l'indique clairement le plan, 4 névés s'échelonnent dans le gouffre. L'Anou Manarf est essentiellement constitué d'un méandre entrecoupé de puits et pénétrable jusqu'à $100 \mathrm{~m}$. Il montre la même structure (Quinif, 1976c).

Nous nous trouvons donc en face de la séquence évolutive suivante:

- Exploitation par la corrosion d'une faille oblique à pendage de l'ordre de 60 à $70^{\circ}$ et élargissement de cette dernière.

- Formation d'un bogaz par recul de la paroi normale de la faille, la paroi surplombante restant pratiquerpent intacte. 


\section{TESSEREFT MESSAOUD}

LAPIAZ DE BOUSSOUIL

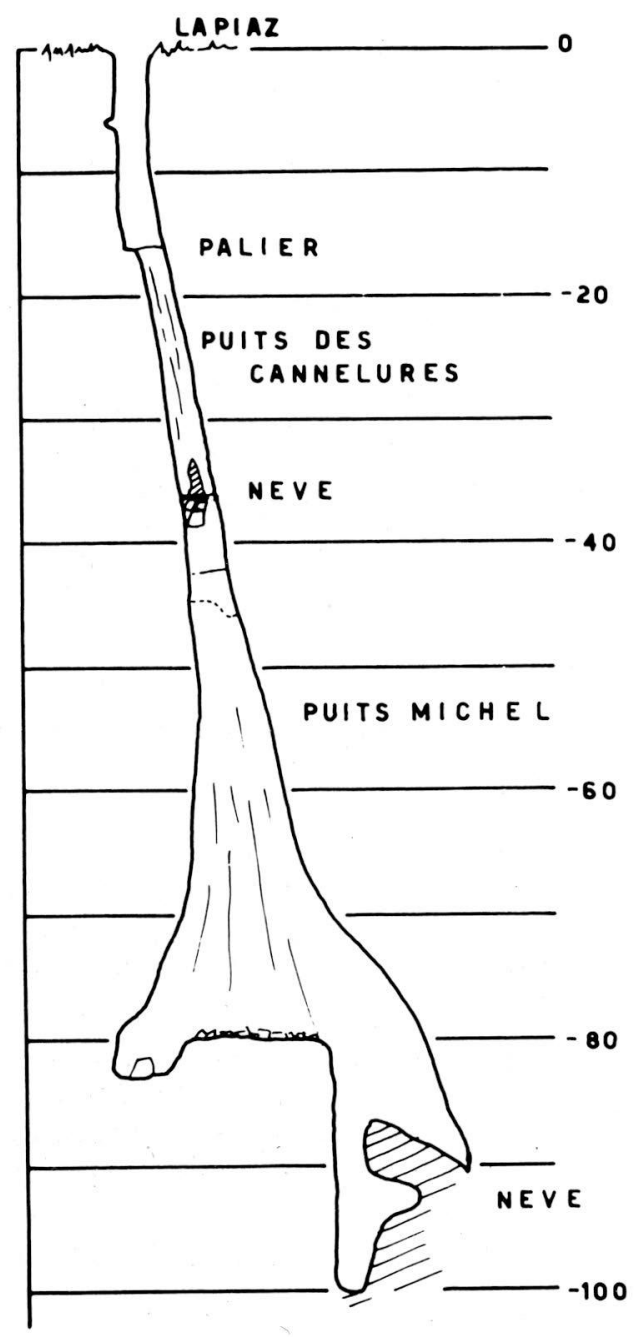

COUPE SUIVANT UN AZIMUT DE $310^{\circ}$ 


\section{TESSEREFT MESSAOUD}

$\begin{array}{r}0 \quad 2 \quad 6810 \mathrm{~m} . \\ \hline\end{array}$
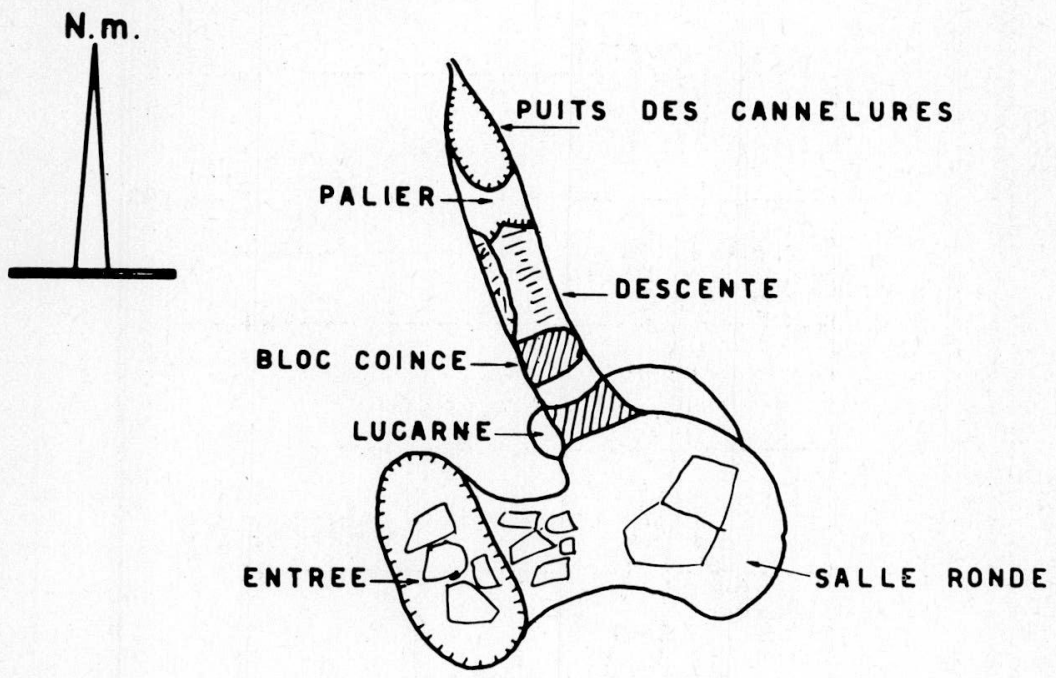

VUE EN PLAN DES PARTIES SUPERIEURES

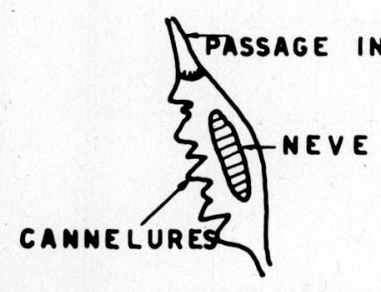

VUE EN PLAN DU

PALIER A -35 .

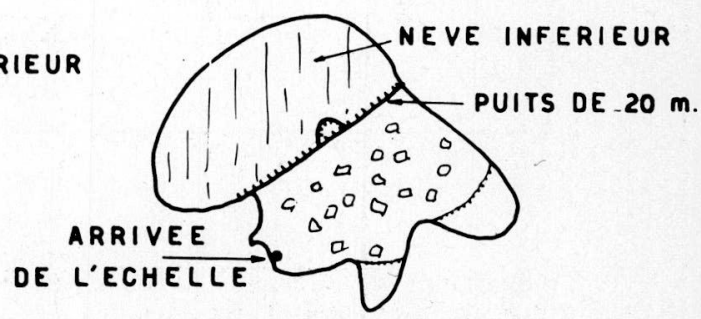

VUE EN PLAN DU

$$
\text { PALIER A }-80 \text {. }
$$

LEYE LE 31 JUILLET 1972 ET LE 24 JUILLET 1973. QUINIFY. BOUCQ MA. E.S.C.

Fig. 9. Plan et coupe de Tessereft Messaoud (lapiaz de la Terga). 


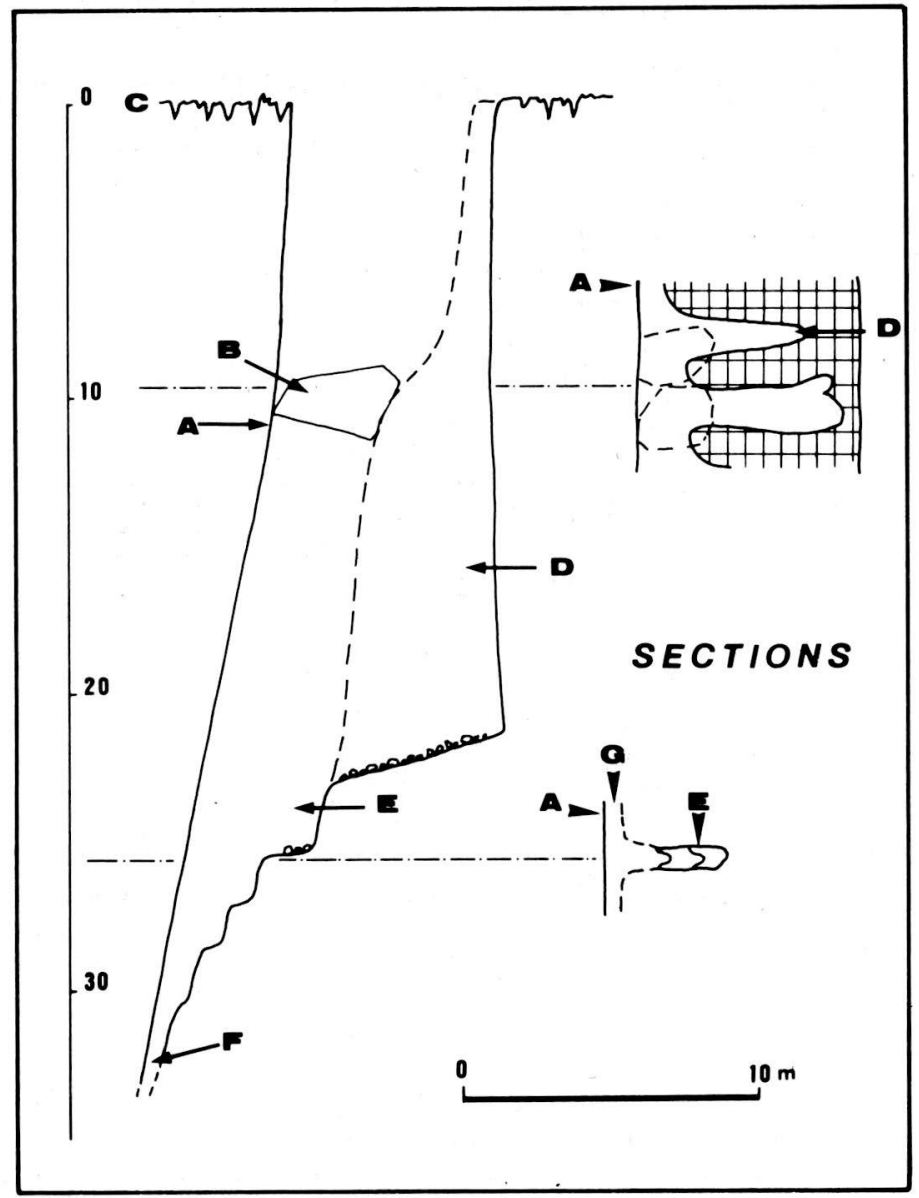

Fig. 10. Coupe dans le «grand bogaz» du lapiaz de la Terga.

A. Paroi frontale surplombante. Il s'agit du mur «inverse» de la faille, à peine touchée par la corrosion.

B. Bloc coïncé.

C. Surface lapiazée.

D. Grande cannelure entaillant la paroi «normale».

E. Méandre entrecoupé de murs de cascade, prolongeant la cannelure aérienne.

F. Endroit où le méandre devient impénétrable.

G. Elargissement creusé au détriment de la faille.

Fig. 11. Coupe de l'Anou Pas-vu-pas-pris. 


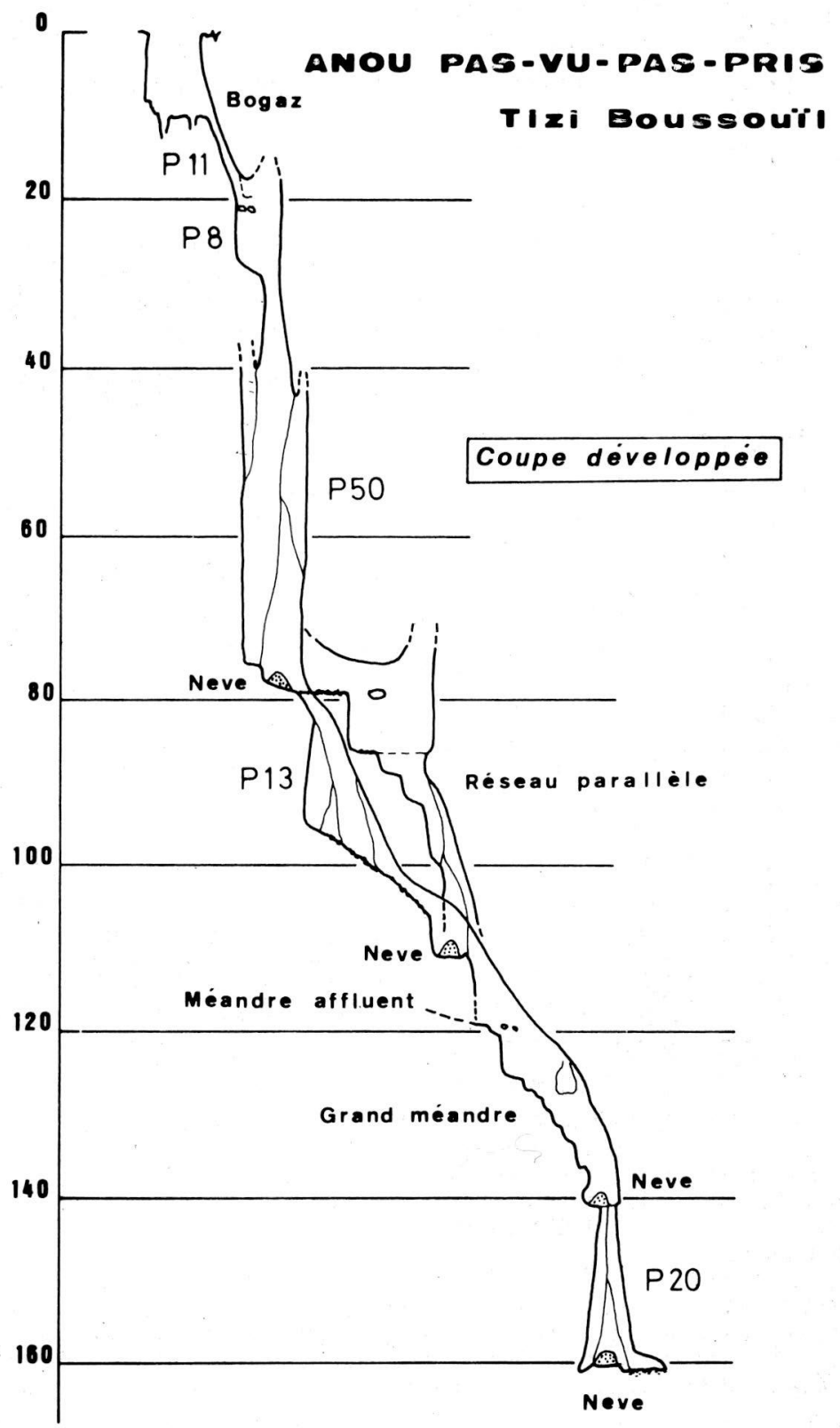

OUINIF Y. ARSO \$-1-75 
- Simultanément, formation de grandes canneleurs de mur sur la paroi normale (forme de surface), se prolongeant par un méandre (forme souterraine). - Intégration des systèmes élémentaires par confluence entre les méandres. Remarquons que le Tessereft Messaoud peut très bien constituer la partie supérieure d'un tel dispositif, le névé inférieur et son plancher empéchant la continuation dans le méandre.

\section{4) Les avens «simples».}

Ce sont de simples puits, sans névé, aboutissant à une profondeur de l'ordre de la trentaine de mètres sur une obstruction par blocs. Ils sont secs, montrant des formes de corrosion comme des dentelles de pierre, de petites cannelures. L'un d'eux, P25 s'ouvront sur la face $\mathrm{N}$ du Thaltat, se creuse dans le Lias supèrieur et est de ce fait très «croulant». Un autre pénètre à $30 \mathrm{~m}$ dans un petit lapiaz de ruissellement sur le flanc S du Gougane.

\section{5) Conclusions sur les avens de lapiaz.}

Nous avons affaire ici à des cavités d'absorption, appartenant à la zone d'alimentation de réseaux haut-alpins, typiques de régions lapiazées. Ils doivent peu à des ruissellements massifs mais bien à des eaux d'infiltrations et de fonte des neiges (superficielles et souterraines). Plus bas, la confluence des méanáres permet - assez rapidement - l'apparition de ruissellements concentrés.

\section{Les émergences de vallées.}

Ce sont les exutoires des grands réseaux. Nous les avons peu explorées. Paradoxalement, elles sont moins accessibles que les cavités sommitales! Ifri Smeddane est une grotte parcourue par un ruisseau, pénétrable par une émergence fossile. Ce ruisseau se remonte à $140 \mathrm{~m}$ au dessus de l'entrée sur plus de $500 \mathrm{~m}$. Il coule dans une galerie parfois vaste, abondamment concrétionnée.

Beaucoup d'émergences sont impénétrables. La «grotte du nez» est l'émergence temporaire du réseau de l'Inker Temdat. On y chemine dans des zones d'éboulis et de galets roulés sans rejoindre un cours permanent.

\section{Grottes de versants.}

\section{1)Généralités.}

Ce dernier type morphologique de cavité acquiert une importance particulère parce qu'il éclaire diverses phases anciennes de l'évolution du Djurdjura. Ce sont des cavités qui s'ouvrent sur les flancs des grandes barres calcaires, face soit aux grandes vallées extérieures à la chaîne (Anou Thinichiguine, Ifri 
Entmgharthe), soit aux vallées subséquentes intérieures à la chaîne (grotte perchée de la Terga, Takouaz Guérissène, Ifri Delhez). Les altitudes des entrées s'étagent entre 1500 et $2000 \mathrm{~m}$.

\section{2) Caractères morphologiques.}

La seule forme représentée significativement dans ces cavités est la galerie, horizontale ou inclinée. Les grottes les plus simples, comme l'Ifri Entmgharte (Fig.12), sont simplement constituées d'une galerie à peu près rectiligne qui s'enfonce perpendiculairement au versant. Les parois et la voûte montrent des concrétions parfois énormes (coulées stalagmitiques, piliers). Les éboulis sont nombreux et parfois gigantesques.

L'Anou Thinichiguine (Fig.13), plus compliqué, apporte d'autres renseignements. La galerie principale est souvent très vaste ( 5 à $10 \mathrm{~m}$ de diamètre). Sur presque toute sa longueur, elle est encombrée d'éboulis qui masquent le sol. Seule la partie inférieure de la galerie laisse apparaître un remplissage fin.

La galerie déclive qui constitue l'entrée est un grand tube de 5 à 7 mètres de diamètre, long de 60 et qui plonge à $40 \mathrm{~m}$ de profondeur. Les parois sont lisses, sauf sur le sol, là où les infiltrations et petits écoulements venant de l'extérieur descendent, laissant des microformes déchiquetées et de petites cannelures. Ces microformes peu importantes ne sont que des retouches.

Nous sommes en présence d'un ancien axe de drainage important. La structure de la grotte suggère une galerie aboutissant à un ancien point d'émergence (vauclusienne par l'actuelle entrée, plus lointaine par la galerie inférieure). Une paroi de la grande galerie montre des coups de gouge indiquant un sens du courant venant du coeur de la montagne vers le versant. Cette observation infirme l'hypothèse d'un aven-perte, de même que l'absence totale de galets roulés.

L'Ifri Delhez, plus à l'ouest, montre également des éboulis dans une galerie beaucoup plus courte parce que tout de suite obstruée par les blocs et le concrétionnement. Nous y avons trouvé des galets roulés d'origine allochtone, notamment des galets de granite. D'après les affleurements de ce dernier, on peut déterminer assez précisément le bassin d'alimentation (col de Tizi n'Chréa). Le courant venait ici des zones internes de la chaîne vers l'extérieur (donc, du S au N).

Le Takouaz Guérissène constitue un cas particulier. En premier lieu, il recèle des formations glaciaires dues à la présence au sommet du Ras Timédouïne et à l'aplomb de la grotte de tesserefts qui assurent une alimentation en neige et en air froid dans la grotte par de conduits verticaux (puits).

Ensuite, la coupe montre deux types de formes qui s'interpénètrent. Les galeries principales sont du type examiné précédemment (vastes, avec éboulis). Elles sont recoupées par des conduits méandres-puits (notamment les nouvelles 


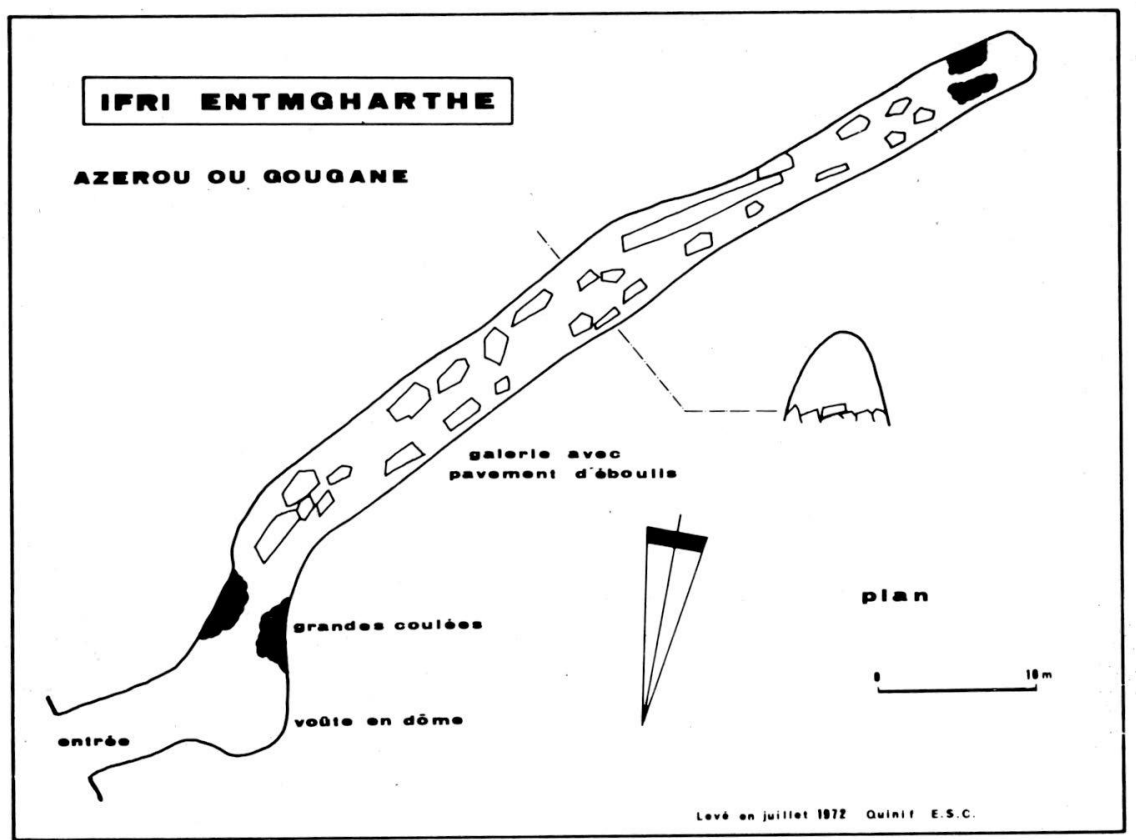

Fig. 12. Ifri Entmgharthe.

régions découvertes en 1977 par une expédition groupant les spéléo-clubs Rosnéen, d'Arles et Lumen Subterra puisque cet ensemble totalise maintenant $170 \mathrm{~m}$ de dénivellation). Ces dernières formes font partie d'un réseau plus récent qui draine les tesserefts et le karst superficiel sus-jacent. Les formations de glace prouvent une circulation actuelle de type alpin trépanant la grotte de versant sans obéir à la logique de cette dernière. Enfin, nous avons trouvé dans la galerie principale du Takouaz des galets roulés de quartz. A cet endroit, ils ne peuvent provenir que de la bande de Trias (grés à dragées de quartz) qui se trouve là en position méridionale et topographiquement inférieure à la grotte. Ils prouvent que l'eau qui circulait dans la grotte à un moment d'hydrologie active de son histoire provenait du S en s'écoulant vers le $\mathrm{N}$. La topographie extérieure était donc très différente. Notons enfin que les grottes de versant ne montrent aucune microforme qui indiquerait un écoulement postérieur plus faible (surcreusement ou recreusement, marmites défoncant d'autres microformes, etc...).

\section{3) Evolution des grottes de versants.}

Ces grottes constituent des drains, parfois importants, fragments d'anciens ré- 
IV. EVOLUTION KARSTIQUE.

A. Vue d'ensemble actuelle.

\section{$\left.1^{\circ}\right)$ Composition d'une unité karstique.}

Schématiquement, un réseau type du Djurdjura se subdivise en 3 zones morphologiquement distinctes.

\section{a) Zone d'alimentation.}

C'est la zone superficielle dans laquelle l'eau gagne rapidement de la profondeur. Elle se caractérise par une pente forte, qui dépend du contexte morphostructural et des quantités d'eau injectées dans le réseau. D'une façon générale, l'alimentation de surface se fait de 4 manières différentes qui correspondent chacune à un type de cavité.

L'alimentation massive par pertes de torrents (Tizi Boussouïl, Haïzer) se fait par des avens-chantoirs, ponors de dépressions fermées de type poljé. Ces derniers s'établissent sur des couches imperméables plus tendres que les calcaires environnants. Les gouffres ont une pente forte $\left(40^{\circ}\right.$ dans l'Inker Temdat et l'Anou Boussouiil) succédant à un cours aérien à pente beaucoup plus faible (quelques degrés avant la perte), avec puits et galeries se suivant en continuité, présence de cuves avec meules, cannelures, surcreusements.

L'alimentation par infiltrations dans les lapiés et dolines ne montre aucune intégration. Seules quelques fissures importantes permettent le développement de cavités qui deviennent vite impénétrables par rétrécissement des conduits. Les avens «simples» correspondent à ce type.

La fonte des névés souterrains fournit une alimentation retardée. Il est possible que la rivière de l'Inker Temdat doive au moins pour une partie à ce type d'alimentation. Ella contribue aussi à élargir les avens tesserefts en fournissant une eau corrosive (eau de fonte à $-5 \mathrm{~m}$ du premier névé du Tessereft Messaoud: $\left.\mathrm{pH}=5, \mathrm{~T}=2.7^{\circ} \mathrm{C},(\mathrm{CaCo})=75 \mathrm{mg} / \mathrm{l}\right)$.

L'alimentation plus brutale par la fonte des neiges contenues dans les bogaz contribue à for̀mer des systèmes cannelures-méandres. Bien qu'il n'existe pratiquement aucun bassin d'alimentation susceptible de fournir un cours d'eau, les grands accidents des lapiés accumulent une quantité de neige telle que la fonte provoque un véritable ruissellement. Néanmoins, ce ruissellement est divisé en de multiples branches non encore intégrées dans la zone d'alimentation superficielle. De ce fait, les cavités sont plus ou moins rapidement impénétrables. Seul l'Anou Pas-vu-pas-pris montre un cas où une relative intégration a commencé. 


\section{b) Zone d'écoulement.}

Elle est quasiment inconnue. Seul l'Inker Temdat nous fournit ce qui constitue sans doute une partie de la zone d'écoulement à forte pente $\left(30^{\circ}\right)$ avec la rivière. Cette pente est moins forte que celle du chantoir, les puits sont de simples crans de descente dans une galerie. Remarquons que la zone d'écoulement de l'Inker Temdat doit nécessairement avoir une pente forte vu le peu de distance horizontale jusqu'à la résurgence. Cette zone d'écoulement (qui diffère aussi du chantoir par la nature et le nombre de microformes, un chimisme des eaux différent), est typique d'un karst de haute montagne (à l'encontre des grottes de versants qui, pourtant, ont une disposition analogue).

\section{c) Zone de sortie.}

Elle est encore quasiment inexplorée. Les émergences sont souvent impénétrables mais l'exploration minutieuse de ces zones devrait donner des résultats intéressants.

\section{$\left.2^{\circ}\right)$ Les grandes unités karstiques.}

a) L'Haïzer.

Massif complexe, il donne naissance à 2 puissantes émergences: les sources de Merkalla au S et Aïn Tinzert au N, toutes deux situées à $900 \mathrm{~m}$ d'altitude. Aucune cavité importante n'est connue sur l'Haïzer, aucune coloration n'a été tentée; il est impossible de délimiter ces deux réseaux.

\section{b) Réseau de l'Inker Temdat.}

Une coloration (Birebent, 1953) a prouvé la communication permanente avec Ansor Arbaïloune. L'origine de la rivière de l'Inker reste inconnue. Le débit des émergences (supérieure à $2 \mathrm{~m}^{3} / \mathrm{sec}$ pour la résurence temporaire) laisse supposer que ce réseau draine le Gougane et la partie W du Thaltat, ainsi peutêtre qu'une partie du Ras Timédouïne.

\section{c) Réseau de la Terga.}

Les eaux perdues dans l'Anou Boussouïl et le lapiaz de la Terga cheminent au sein de la bande calcaire Terga Irouienne - Adrar Timésouïne pour ressortir à Acif-el-Hammam, donc sur le versant $\mathrm{N}$ de la chaîne, $1000 \mathrm{~m}$ plus bas que l'entrée de l'Anou, là où l'oued el Hamman qui prend naissance dans le bassin subséquent d'Aït Ouabane sort par une gorge épigénétique en perçant la barre 
calcaire. Lors des crues, la vitesse horaire de l'eau est de $44 \mathrm{~m}$ ce qui est élevé. Le réseau est bien intégré.

\section{d) Le Tamgout de Lala Khedidja.}

Très peu karstifié, ce massif possède des émergences importantes à Ansor Aberkane et Ansor Lekalt.

\section{$\left.3^{\circ}\right)$ Caractères hydrogéologiques.}

a) Pertes massives.

L'alimentation est très localisée ce qui a d'ailleurs généré une cavité importante: ensemble puits-galeries à forte pente qui conduit rapidement l'eau dans les profondeurs du massif. Cet apport d'eau est relativement ponctuel: fontes des neiges, suite d'orages violents. Il donne naissance à de forts débits aux émergences durant des périodes courtes. Les eaux de l'Inker Temdat résurgent de façon permanente à Ansor Arbaïloune avec un débit d'étiage compris entre 120 et $160 \mathrm{l} / \mathrm{sec}$ tandis que la résurgence supérieure ne fonctionne qu'en crues en débitant alors 1 à $2 \mathrm{~m}^{3} / \mathrm{sec}$. D'autre part, une coloration dans le réseau de la Terga (Birebent, 1953) lors d'une fonte des neiges peu importante a montré que la vitesse horaire moyenne était de $44 \mathrm{~m}$.

\section{b) Fonte des neiges de surface,}

En dehors des surfaces imperméables conduisant les eaux de fonte vers les pertes ou en dehors des aires kartiques, la neige peut néanmoins être emmagasinée en assez grandes quantités, notamment dans les grands bogaz. Leur fonte fournit des débits comparables à celui des pertes massives, bien que moins abondants. Ils restent limités dans le temps et soutiennent essentiellement des débits de crues aux émergences. Remarquons à ce sujet que, lors de la coloration du réseau de la Terga, le débit à l'Anou Boussouïl était de $10 \mathrm{l} / \mathrm{sec}$ alors qu'il était de $1 \mathrm{~m}^{3} / \mathrm{sec}$ à la résurgence. En sachant qu'aucune autre perte massive n'alimente ce réseau, l'apport des eaux de fonte des neiges de surface est donc considérable.

\section{c) Fonte des névés souterrains.}

Cette alimentation s'étale sur tout l'été et est donc essentiellement différente. C'est donc un apport économiquement primordial car il fournit de l'eau durant la saison sèche. Il est bien connu des villageois du pied de la montagne 
qu'un hiver à faible enneigement est très défavorable au débit d'été des sources. $\mathrm{Vu}$ le peu d'importance des zones noyées, on en déduit le rôle des névés souterraines (constaté sur place grâce aux explorations de tesserefts).

\section{d) Infiltrations généralisées.}

Elles concernent les précipitations qui se perdent dans les lapiés et les dolines. Leur effet est mal appréhendé et leur étude exige l'analyse des hydrogrammes des sources en relation avec le climat.

\section{e) Condensation souterraine.}

Favorisées par le contraste entre les températures des cavités (de 0 à $5^{\circ} \mathrm{C}$ dans les gouffres) et celles de l'extérieur, ainsi que par les circulations importantes d'air dans ces cavités, elles contribuent incontestablement à l'alimentation des émergences. Les parois des avens sont toujours humides, parfois constellées de gouttes d'eau.

\section{B. Le karst actuel et l'évolution passée.}

Le Karst actuellement actif du Djurdjura, dans sa zone d'alimentation, est évidemment sous le contrôle de son contexte structural, morphologique et climatique. Le Djurdjura est une montagne alpine. Les surfaces d'enfouissement sont portées à haute altitude, ce qui induit ici un climat de type nival. La tectonique alpine est la cause de structures complexes de charriages, redressées à la verticale. Autre conséquence de l'evolution structurale, les crêtes dominent de plus de $1000 \mathrm{~m}$ le pays environnant.

De ces caractères dépendent les caractéristiques du karst actuellement actif. Le climat de type nival (renforcé lors de certaines périodes quaternaires, Quinif, 1976a) permet une morphologie superficielle de type haut-alpin: lapiaz, dolines, tesserefts. Elle a une importance primordiale sur les différents types d'écoulements souterrains. L'intense tectonisation, nous en avons vu aussi quantité d'exemples, oriente nettement la structure et la morphologie des cavités (systèmes cannelures-méandres, Anou Boussouïl). Le relief de la montagne fournit d'importantes dénivelées. Jointes à la fracturation, elles permettent aux eaux souterraines de s'enfouir rapidement en profondeur. De plus, cette topographie dominante a pour conséquence d'induire dans les masses rocheuses un régime mécanique de détente quasi-généralisé. De ce point de vue, nous avons ici affaire à un karst de montagne sous une forme particulièrement pure (Renault, 1967).

Les lapiés, dolines, poljés, en connection morphologique avec des cavités tel- 


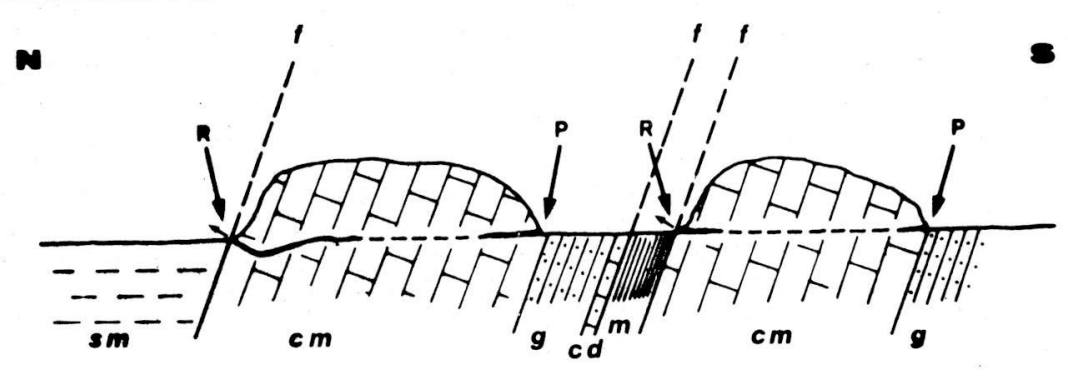

Fig 14 a.

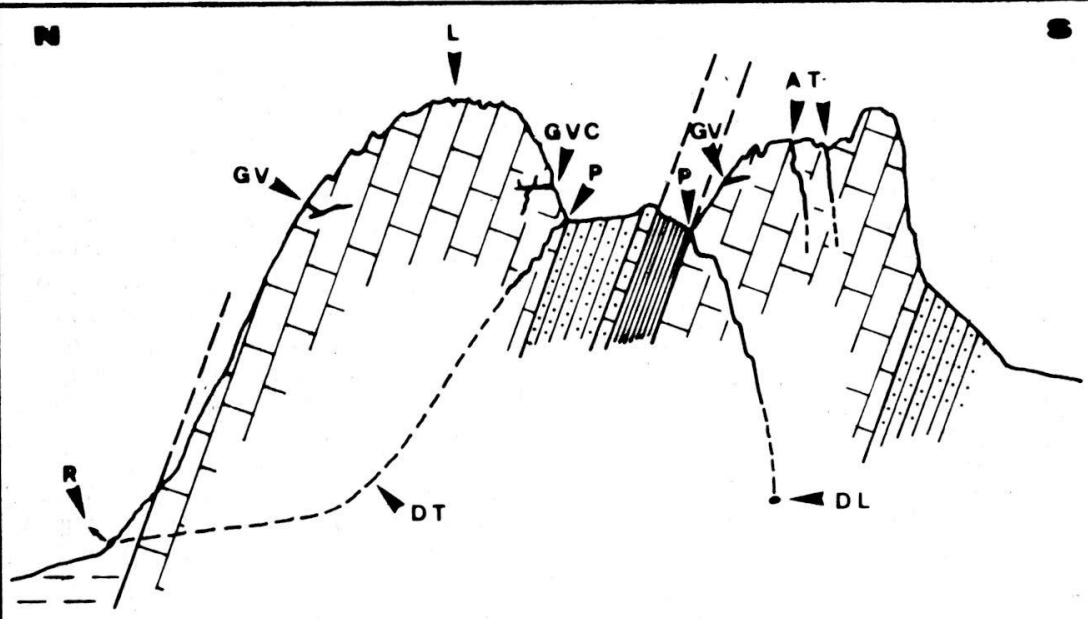

Fig $14 \mathrm{~b}$.
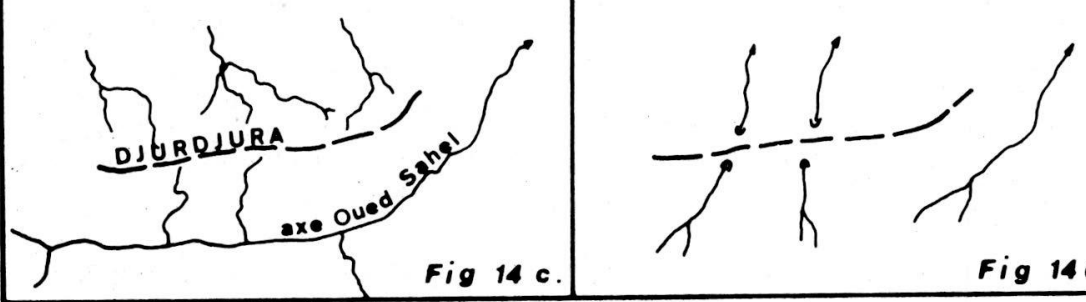

Fig $14 d$. 
Fig. 14. Séquence évolutive de principe du Djurdjura. Ces coupes n'ont d'autre ambition que d'être explicatives. Ni les distances horizontales, ni les altitudes, ni les détails de la géologie ne sont respectés. De plus, nous avons groupé plusieurs phénomènes qui, en fait, se trouvent en des endroits éloignés les une des autres.

Fig.14a. Coupe N-S lorque les grottes des versants étaient hydrologiquement actives. On voit qu'elles constituent des grottes-couloirs canalisant des rivières venant du $\mathrm{S}$ au travers des barrières calcaires naissantes du Djurdjura. Ces dernières ne s'individualisent pas très bien dans le paysage. De plus, leur altitude faible ne permet pas à un environnement montagnard de d'installer. Nous avons affaire à un relief moins contrasté. En $\mathbf{P}$ nous avons les pertes et en $\mathrm{R}$ les résurgences. Les traits plus gras symbolisent les grottes. Dans cette figure, nous avons schématisé deux écailles avec la sequence Trias-Lias, les contacts anormaux (f) et des marnes paléogènes également en position anormale.

s.m.: socle métamorphique kabyle.

c.m.: calcaires massifs (Lias).

g.: grés (Trias supérieur).

c.d.: calcaires dolomitiques (Trias moyen).

m.: marnes (paléogène).

Remarquons encore que cette disposition hydrogéologique explique les faits observés dans les grottes de versant, tels que la nature des galets roulés trouvés dans ces grottes et l'allure des coups de gouge.

Fig. 14b. Même coupe que celle de la Fig.13a, mais se situant à l'époque actuelle. La néotectonique a individualisé le Djurdjura en temps que montagne avec ses grandes barrières calcaires. La surrection permet aux parties sommitales (calcaires) d'atteindre des altitudes telles qu'elles subissent d'un karst haut-alpin avec ses surfaces lapiazées (L.), ses avens-tesserefts (A.T.), etc. De plus, le réseau hydrographique s'est fondamentalement modifié. Alors que les anciens axes de drainage souterrain se trouvent démantelés et portés en altitude où ils subsistent en temps que grottes de versants (G.V.), les écoulements actuels sont centrés sur la montagne. La coupe montre un polje (Tizi Boussouïl), d'origine structurale (établi sur des assises imperméables) avec ses deux ponors (p.) suivis de gouffres-chantoirs. On reconnaît les deux types de réseaux. Celui de gauche est transversal (par exemple le réseau de l'Inker Temdat) parce que l'axe de drainage souterrain (D.T.) est perpendiculaire à l'écaille de calcaire. Celui de droite est longitudinal (comme celui de l'Anou Boussouil) parce que l'axe de drainage (D.L. vu ici en section) s'allonge dans l'axe de la barre calcaire. En G.V.C.,nous avons visualisé une grotte de versant complexe car reprise dans un réseau actuel (exemple du Takouaz Guérissène).

Fig. 14c. Allure actuelle de réseau hydrographique. On voit qu'il est partagé par le Djurdjura. Les oueds du N alimentent l'Oued Sebaou tandis que les écoulements S sont drainés par le sillon Oued Sahel-Oued Soummam. Notons que les principaux oueds dérivent d'une ou plusieurs émergences karstiques.

Fig. 14d. Reconstitution de l'allure du réseau hydrographique à l'époque de la coupe de la Fig.13a. Les oueds venant du S buttent contre les barrières calcaires naissantes du Djurdjura, les traversent dans des grottes et continuent vers le N. Notons que la surrection du Djurdjura, la subsidence de l'axe de l'Oued Soummam et l'érosion régressive de l'Oued Sahel s'additionnent pour aboutir à la situation actuelle.

les que les avens-chantoirs, avens-tesserefts, systèmes cannelures-méandres, sont donc comptemporains de l'environnement morpho-structural de type haut-alpin tel que nous le constatons aujourd'hui. Les grottes de versants ne s'intègrent pas dans ce contexte. Elles correspondent à des circulations transversales aux barres calcaires dans un relief différent, moins montagnard, datant d'avant ou du début de la surrection du Djurdjura. Ces circulations drainaient les eaux du $\mathrm{S}$ vers le $\mathrm{N}$ à travers des barrières calcaires naissantes. Cela nous ramène à une époque antérieure à l'axe de drainage Oued Sahel - Oued 
Soummam, axe ayant pris naissance à cause du soulèvement trop rapide du Djurdjura (antécédence n'ayant pu se réaliser) et du mouvement de subsidence dans le sillon de la Soumman.

\section{CONCLUSIONS.}

Le Djurdjura posséde un karst de type haut-alpin remarquable à plus d'un titre: morphologie de surface, souterraine, circulations, etc...Nous achevons ici la description de ses principaux caractères, commencée ailleurs (Quinif, 1976a, 1977), avec l'aspect morpho-hydrogéologique des cavités. Ces dernières se divisent en 2 classes fondamentalement différentes: les avens et grottes appartenant aux actuels réseaux actifs (avens-chantoirs, avens-tesserefts, système cannelures-méandres, grottes-émergences) et les grottes de versants témoins d'anciens réseaux à présent démantelés, parfois repris en partie par des circulations actuelles. Les cavités du premier type se caractérisent par une structure, une morphologie et des dépôts en accord avec leur fonction hydrogéologique, leur contexte structural, topographie et climatique. Les secondes sont déconnectées de ce contexte et témoignent d'un environnement profondément différent.

Il faut remarquer que ceci en est pour le moment au stade d'hypothèses, probables certes mais non vérifiées. Elles ont le mérite d'indiquer une voie de recherche originale, à savoir la contribution de la spéléologie à l'histoire morpho-structurale d'une région. Il manque toute une série de preuves, comme les études précises et détaillées de sédiments à la fois dans les réseaux alpins et les grottes de versants, avec datation, analyse pétrographique (abordée ici et prouvant la fécondité de telles études) et sédimentologiques, analyse polinique afin de déterminer les contextes paléo-climatiques. Cela constitue une phase ultérieure dont l'étude actuelle ne constitue qu'un préambule.

\section{RÉSUMÉ}

Dans le cadre de l'Afrique du Nord, le karst du Djurdjura acquiert une importance particulière car il présente des caractères haut-alpins. Après avoir abordé dans d'autres articles la morphologie superficielle et des aspects purement spéléologiques, nous décrivons ici les cavités des points de vue morphologie, hydrogéologie qualitative et sédiments. Nous les replaçons dans leur contexte morpho-structual ce qui nous permet de faire des distinctions entre différents types de cavités. D'une part, nous avons les avens et résurgences caractérisent le karst actuel haut-alpin (avenspertes, avens de lapiaz, grottes-résurgences); de l'autre, des grottes de versants, sèches, déconnectées du contexte actuel, se rattachent à une phase de karstification antérieure. De la synthèse de ces éléments,nous essayons de mettre en évidence la possibilité d'utiliser les données tirées du karst dans la reconstitution de l'évolution morpho-structurale d'une région. 


\section{BIBLIOGRAPHIE}

BÉLIN A., MARICHAL R., SACCARDY L., TROULLIER J., VERGÈ H., 1941 - Notes spéléologiques sur le Djurdjura. Bull. Serv. Carte Géol. d'Algérie, $3^{\mathrm{e}}$ série, $7^{\mathrm{e}}$ fasc.

BÉLIN A., 1948 - Trois abîmes du Djurdjura. Ann. Spéléol., III: 145-152.

BIREBENT J., 1948 - Explorations souterraines en Algérie. Ann. Spéléol., III (2-3):49-144.

BIREBENT J., 1953 - Explorations souterraines en Algérie. Ann. Spéléol. III (9-62):70-127.

CHAUMONT M., PAQUIN C., 1971 - Carte pluviométrique de l'Algérie. Soc. Hist. nat. d'Afrique du Nord.

COIFFAIT P.E., QUINIF Y., VILA J.M., 1975 - Synthèse sur l'histoire géologique et les karstifications des séries néritiques constantinoises. Ann. Spéléol. 30, (4):619-627.

COUTELLE A., 1976 - Flyschs externes et unités telliennes du flanc sud du Djurdjura. Présentation d'un modèle d'évolution tectogénique de la Grande Kabylie. Bull. Soc. Géol. Fr., 7e série, XVIII (5):1337-1345.

COUTELLE A., 1977 - Les grands traits stratigraphiques et structuraux du Djurdjura. $A S A C$. 2:15-18.

FLANDRIN J., 1952 - La chaîne du Djurdjura. XIXe congrès géologique international, Monographies régionales, $\mathrm{I}^{\mathrm{e}}$ série, n. 19. Alger.

QUINIF Y., 1973 - Compte rendu des expéditions de l'Equipe Spéléo du Centre en Algérie (19711972). Speleologia Belgica, n. 1:11-13; n. 2:12-20.

QUINIF Y., 1975 - L'Anou Inker Temdat. Spelunca. n. 1:21-25.

QUINIF Y., 1976a - Contribution à l'étude morphologique des karsts algériens de type hautalpin. Rev. Géogr. Phys. Dyn., XVIII (I):5-18.

QUINIF Y., 1976b - Nouvelles explorations en Algérie (1975). Spelunca, n. 1:13-16.

QUINIF Y., 1976c - Un type particulier d'écoulement karstique en zone d'alimentation à partir de lapiaz de type haut-alpin (Djurdjura, Algérie). Ann. Spéléol., 31:69-73.

QUINIF Y., 1977 - Quelques aspects du karst du Djurdjura (Algérie): les zones de Tizi Boussouïl et du Djebel Haïzer. Leurs dépressions fermées. Rev. Géogr. Phys. Géol. Dyn., XIX (2):137-148. RENAULT P., 1967 - Contribution à l'étude des actions mécaniques et sédimentologiques dans la spéléologenèse. Ann. Spéléol., 22:209-267.

RENAULT P., 1968 - Contribution à l'étude des actions mécaniques et sédimentologiques dans la spéléologenèse. Ann. Spéléol., 23:259-596.

SELTZER P., 1946 - Le climat de l'Algérie. Université d'Alger. Institut de météorologie, Alger. TROMBE F., 1952 - Traité de spéléologie. Payot Ed., Paris, 376 p., 120 fig. 